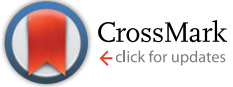

Received 14th September 2016 Accepted 26th October 2016

DOI: $10.1039 / c 6 t a 07984 a$

www.rsc.org/MaterialsA
Cite this: J. Mater. Chem. A, 2017, 5, 31

\section{A review on special wettability textiles: theoretical models, fabrication technologies and multifunctional applications}

\begin{abstract}
Shuhui Li, ${ }^{a}$ Jianying Huang, ${ }^{a}$ Zhong Chen, ${ }^{b}$ Guoqiang Chen ${ }^{a}$ and Yuekun Lai ${ }^{\star a c}$
Inspired by the superhydrophobic lotus surface in nature, special wettability has attracted a lot of interest and attention in both academia and industry. In this review, theoretical models and fabrication strategies of superhydrophobic textiles have been discussed in detail. The strategies for constructing fabric surfaces with an anti-wetting property are categorized and discussed based on the morphology of particles coated on the textile fibre. Such special wettability textile surfaces are demonstrated with selfcleaning, oil/water separation, self-healing, UV-blocking, photocatalytic, anti-bacterial, and flameretardant performances. Correspondingly, potential applications have been illustrated for self-cleaning, oil/water separation, asymmetric/anisotropic wetting janus fabric, microfluidic manipulation, and microtemplates for patterning. In each section, representative studies are highlighted with emphasis on the special wetting ability and other relevant properties. Finally, the difficulties and challenges for practical application were briefly discussed.
\end{abstract}

\section{Introduction}

An extremely high water contact angle of nearly $180^{\circ}$ was first reported by Ollivier in 1907. Such a super-antiwetting surface

${ }^{a}$ National Engineering Laboratory for Modern Silk, College of Textile and Clothing Engineering, Soochow University, Suzhou 215123, China. E-mail: yklai@suda.edu.cn ${ }^{b}$ School of Materials Science and Engineering, Nanyang Technological University, 50 Nanyang Avenue, Singapore 639798, Singapore

${ }^{c}$ Research Center of Cooperative Innovation for Functional Organic/Polymer Material Micro/Nanofabrication, Soochow University, Suzhou 215123, China was fabricated via coating a substrate using soot. ${ }^{1}$ The superhydrophobic surfaces with a high static water contact angle $\left(>150^{\circ}\right)$ and low sliding angle $\left(<10^{\circ}\right)$ had not received much attention until the report of the "lotus effect" mechanism by Barthlott and Neinhuis in 1997. ${ }^{2}$ This unique property was attributed to the combination of a waxy layer with a low surface energy and a rough structure with protrusions on lotus leaves (Fig. 1). ${ }^{3,4}$ Since then, research interests on superhydrophobicity have grown tremendously, with numerous studies devoted to mimicking natural plants,

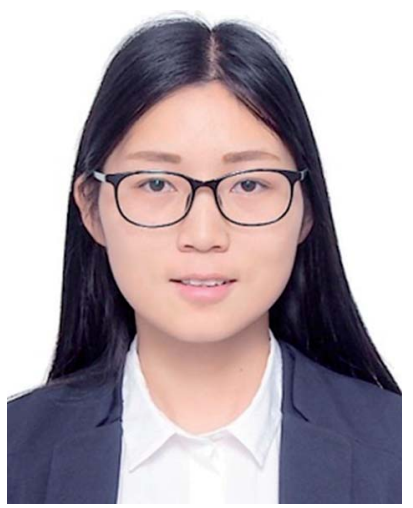

Shuhui Li joined Prof. Lai's group in College of Textile and Clothing Engineering at Soochow University to pursue her Master's degree in 2013. She continued to pursue her Ph.D. degree at Soochow University from 2015. Her current scientific interests are devoted to fabricating special wettability and multi-functional textile surfaces for applying in self-cleaning, oilwater separation, UV shielding and anti-bacterial materials.

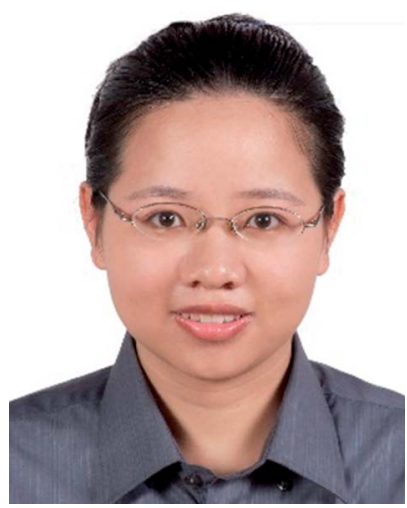

Jianying Huang received her Ph.D. degree in 2007 from the Department of Materials, Xiamen University. During 20072011, she worked as an assistant professor at Fujian Institute of Research on the Structure of Matter. After that, she moved to Muenster University as a visiting scholar. She is currently an associate professor at National Engineering Laboratory for Modern Silk, and School of Textile and Clothing Engineering in Soochow University since 2013. Her research interests focus on the bio-inspired intelligent textile surfaces with special wettability, ATRP and click-chemistry reactions. 
animals and creatures. Great efforts were devoted to the understanding of the relationship between the structures and special wettability of natural creatures and fabricating artificial superhydrophobic surfaces. Such superhydrophobic characteristics have been widely explored for self-cleaning, anti-fogging/frosting, oil/water separation, and anti-bioadhesion applications.

Recently, different micro/nanoscale binary structured superhydrophobic surfaces with a high static water contact angle and low hysteresis have opened up new possibilities of applications in industrial and biological fields. ${ }^{5-10}$ Textiles, for example, are intrinsically porous, rough, flexible and hydrophilic. At the as-fabricated state, they absorb both water and oil. ${ }^{11,12}$ For apparent reasons, it is desirable to modify the hydrophilic textile with low surface energy materials in order to overcome the intrinsic weakness for a number of applications.

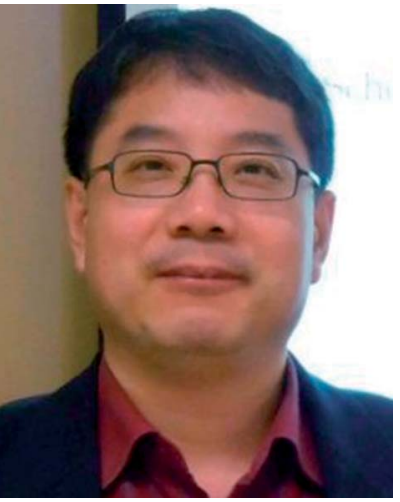

Zhong Chen obtained his Ph.D. in 1997 at the University of Reading, U.K. After completing the study, he joined the newly established Institute of Materials Research and Engineering, a national research institute funded by Singapore government. In March 2000, he moved to Nanyang Technological University as an Assistant Professor, and has since been promoted to Associate Professor and Professor in the School of Materials Science and Engineering. Prof. Chen's research interests include Surface Engineering, Thin Films \& Nanostructured Materials, and Mechanical Behaviour of Materials. He is an author of over 200 SCI journal papers, 5 book chapters and 5 granted patents.

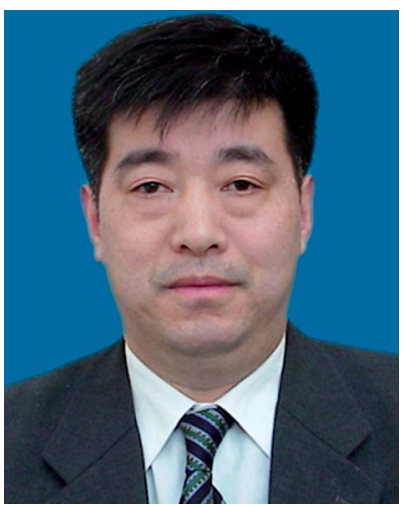

Guoqiang Chen is a professor in the College of Textile and Clothing Engineering at Soochow University (China). He received his Ph.D. degree in textile chemistry and dyeing \& finishing engineering at Donghua University (China). He pursues research and development of textile chemicals and functional textiles. He has published more than 250 papers. He serves as Editor-In-Chief of Modern Silk Science and Technology. He has received several prizes (State Science and Technology Awards, Mulberry and Flax Prize of Hong Kong). He is vice president of the China Silk Association.

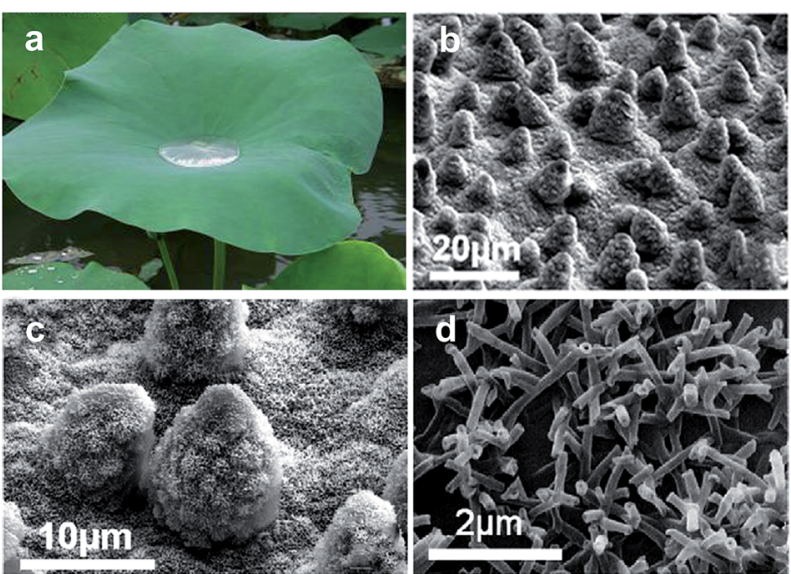

Fig. 1 Lotus leaves in nature: self-cleaning behaviour (a) and the related microstructures as observed by scanning electron microscopy (b), protrusions (c) and the wax tubules on them (d). (Reprinted from ref. 4 with permission).

It would require unique micro/nanostructures inspired by nature to enable special wettability for the textile industry. Cloths with oil-water separation are valuable to combat the problems caused by oil spill accidents. ${ }^{\mathbf{1 3 4}}$ Moreover, other novel multifunctional applications for superhydrophobic textiles started to merge, including UV-blocking, photocatalytic, flame-retardant, asymmetric superhydrophobic/ superhydrophilic, and stimuli-responsive. These represent potentially high value-added textiles that can be realised by relatively simple chemical treatment. However, there are potential issues for the health and safety of the workers and consumers during processing and usage. Thus, attention should be paid to research on environmentally friendly preparation methods, as well as durability and mechanical stability of the textiles.

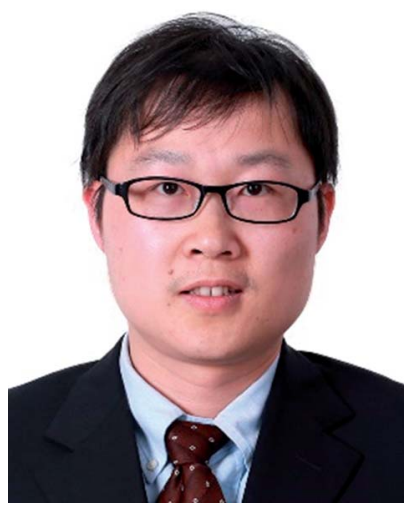

Yuekun Lai received his Ph.D. degree from the Department of Chemistry, Xiamen University. During 2009-2011, he worked as a research fellow at the School of Materials Science and Engineering, Nanyang Technological University, Singapore. After that, he moved to Muenster University with Prof. Harald Fuchs and Prof. Lifeng Chi under the support of the Humboldt Foundation Scholarship of Germany. He is currently a professor at National Engineering Laboratory for Modern Silk, and School of Textile and Clothing Engineering in Soochow University since 2013. His research interests focus on the bio-inspired intelligent surfaces with special wettability, and energy \& environmental materials. 


\section{Characterization of superhydrophobic surfaces}

Generally, the surface with static water angle $\theta_{\mathrm{CA}}>150^{\circ}$ and sliding angle $\theta_{\mathrm{SA}}<10^{\circ}$ is defined as superhydrophobic. However, the definitions of the hydrophobic state are divided into two camps. One believe that the solid surface with a contact angle $\theta_{\mathrm{CA}}>90^{\circ}$ was considered as hydrophobic according to Young' equation. The other view by Volger $e t$ al. suggested that a CA of $65^{\circ}$ divides solid materials into hydrophobic and hydrophilic using a surface force apparatus supported by ancillary techniques. ${ }^{15}$ Specifically, attractive forces appeared when two planes exhibited a water $\mathrm{CA}>65^{\circ}$. In contrast, repulsive forces were detected between surfaces with $\theta<65^{\circ}$. This result clearly demonstrates that the new division of hydrophilicity and hydrophobicity should be $65^{\circ}$ rather than $90^{\circ}$ when considering the chemical and structural states of water droplets. Similar to this research, Jiang et al. found that an angle of $62.7^{\circ}$ could distinguish hydrophilicity and hydrophobicity after investigating the apparent and intrinsic CA of many polymeric materials. ${ }^{16}$ Therefore, the two general routes to fabricate superhydrophobic surfaces can be summarized as follows: constructing appropriate roughness on the material surface or chemically modifying the material surface to be hydrophobic with a contact angle $>65^{\circ}$.

The simplest method to evaluate a superhydrophobic surface is by visualization with eye. When a flow of water is applied on the substrate surface, an intuitional wetting behaviour and a self-cleaning phenomenon are observed. However, it is essential to characterize the superhydrophobic property quantitatively with precise determination of the static water contact angle (WCA) and contact angle hysteresis (CAH). Notably, superhydrophobic surfaces with a similar water contact angle $\theta_{\mathrm{CA}}>150^{\circ}$ can exhibit completely different contact angle hysteresis. ${ }^{17,18}$ To distinguish among the different superhydrophobic states, five typical wetting states have been defined (Fig. 2a-e):19 totally wetting superhydrophobic surfaces in the Wenzel state, totally air-supporting superhydrophobic surfaces in the Cassie state, the metastable state between the Wenzel and Cassie states (including a "petal" state), surfaces in the micro/ nanostructured two-tier "lotus" state, and a partially wetting "gecko" state. Nature also provides several examples of anisotropic superwettability. For example, water droplets easily roll along the direction parallel to the rice leaf edge but not in the perpendicular direction..$^{20}$ Similar to rice leaves, butterfly wings

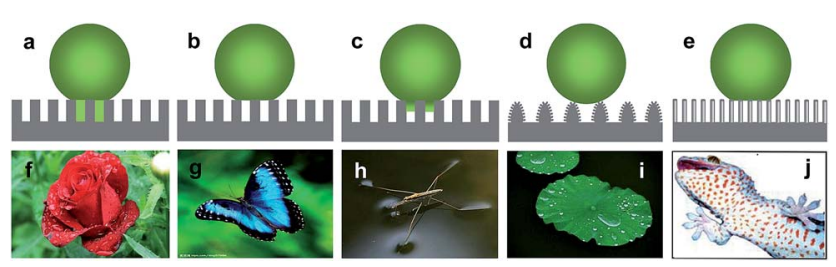

Fig. 2 Five typical cases for anti-wetting surfaces (a: Wenzel state; b: Cassie state; c: Wenzel-Cassie state; d: "lotus" state; e: "gecko" state) and several common phenomena for anti-wetting in nature (f: petal; $g$ : butterfly; h: strider; i: lotus leaf; j: gecko).
(Fig. 2g) also exhibit anisotropic rolling/pinning superhydrophobic states. ${ }^{21}$ In addition to these low-adhesion superhydrophobic examples, some organisms also provide examples of high-adhesion superhydrophobic properties, such as gecko feet (Fig. 2j). ${ }^{22-24}$

Generally, there are three important wetting theories to explain the surface wettability: the Young model, Wenzel model and Cassie-Baxter model. The Young equation assumes that the surface is totally smooth and the static contact angle is determined by the interfacial energies between the solid-vapour phase, solid-liquid phase, and liquid-vapour phase. However, most solid surfaces in reality are rough and this has put a limitation on Young's model in explaining the surface wettability behaviour. Wenzel revised the Young equation and suggested that the real contact area increases on a roughened surface. In the Wenzel state, the liquid is considered to be in complete contact with the rough solid surface so it is difficult for the water droplet to move and roll off the surface due to the larger contact area and stronger adhesion. Subsequently, Cassie and Baxter proposed a heterogeneous contact model where the liquid is on top of the rough surface with air trapped in between. Therefore, a water droplet is easy to move and roll off the surface, which is described as a "slippy" surface. ${ }^{25}$ Theoretical analysis has confirmed that the Wenzel state corresponds to a lower energy level. ${ }^{26,27}$ The presence of surface energy between these two states may prevent the spontaneous transition from the Cassie state to Wenzel state, but it is confirmed that the two models can transit from one to the other under specific conditions.

\subsection{Static water contact angle}

Thomas Young was the first to consider the contact angle of a water droplet on a completely smooth substrate in relation to the interfacial energies acting between solid-liquid $\left(\gamma_{\mathrm{sl}}\right)$, solidvapor $\left(\gamma_{\mathrm{sv}}\right)$ and liquid-vapour $\left(\gamma_{\mathrm{lv}}\right)$ interfaces in the well-known Young's equation:

$$
\cos \theta_{\mathrm{ca}}=\frac{\gamma_{\mathrm{sl}}-\gamma_{\mathrm{sv}}}{\gamma_{\mathrm{lv}}}
$$

The equation assumed an ideal state that the surfaces are chemically homogeneous, atomically smooth and the surface wettability is dependent on the chemical composition at the interfaces. However, even for the low surface energy material (e.g., C9 perfluorocarbon) modified substrate, the static water contact angle can only reach $105^{\circ}-118^{\circ},{ }^{28}$ which is below the defined superhydrophobic contact angle of $150^{\circ}$. Therefore, the surface roughness is very necessary to fabricate superhydrophobic surfaces. Two different wetting models, including the Wenzel model and the Cassie model, are developed to explain the wetting behaviour of the roughness substrate since the actual surface is not absolutely smooth. In the textile field, a commonly employed strategy is to construct nano-microroughness using particles and to modify the surface using a low surface energy material. Although many literature studies reported successful attempts for superhydrophobic anti-wetting textile through a novel one-step deposition of 
a fluoroalkylsilane polymer, concerns exist over the stability and durability, as well as the potential toxicity of fluorine-containing molecules to the environment.

Both the Wenzel model and Cassie model clarified the mechanism of wettability on the roughness surface and demonstrated that surface roughness and low surface energy are two equally critical factors for constructing the extreme wetting surface states. The Wenzel and Cassie model equations are given in eqn (2) and (3) respectively:

$$
\begin{gathered}
\cos \theta_{\mathrm{w}}=\gamma \cos \theta_{\mathrm{e}} \\
\cos \theta_{\mathrm{r}}=f_{\mathrm{s}}(\cos \theta+1)-1
\end{gathered}
$$

In the Wenzel state, a liquid droplet is in complete contact with the substrate and the static contact angle is proportional to the contact angle on the flat surface and the surface roughness factor $(r)$ (eqn (2)). The surface roughness factor $(r)$ is defined as the ratio of the actual contact area and the projected surface area. In this situation, the surface roughness factor is always larger than one; thus for a hydrophobic surface, the hydrophobic property improves (i.e. the actual contact angle increases) with increasing surface roughness. Moreover, the surface roughness is also beneficial to improve wetting by making the contact angle smaller for a hydrophilic state (WCA < $90^{\circ}$ ). When water is not able to completely wet the rough substrate surface due to protrusions and recessed areas with trapped air in between, the Cassie model (eqn (3)) becomes applicable. The Cassie model is based on water contact with a composite surface of the solid and air that is trapped into the microgrooves of the rough surface. In eqn (3), $f_{\mathrm{s}}$ is the fractional contact area. Based on this equation, the contact angle increases with increasing fractional air-liquid contact defined by $\left(1-f_{\mathrm{s}}\right)$. Therefore, to obtain a superhydrophobic surface, it is very necessary to design fractal dimensions of the rough surface for enlarging the contact area of liquid and air. So altering the surface free energy or fabricating the roughness surface structure or both can achieve a superhydrophobic surface.

\subsection{Contact angle hysteresis}

As stated earlier, the superhydrophobic surface exhibits various dynamic characteristics and can be potentially applied for various applications including self-cleaning, anti-corrosion, anti-biology adhesion, and snow-pinning prevention. The criterion to judge a superhydrophobic surface is not only by the static liquid contact angle, but also the dynamic contact angle. For a dynamic water droplet, there are two contact angles, the so-called advancing contact angle $\left(\theta_{\text {Adv }}\right)$ and the receding contact angle $\left(\theta_{\text {Rec }}\right)$. The difference between them is defined as contact angle hysteresis (CAH). Generally speaking, a true superhydrophobic surface means not only a high static contact angle, but also a low contact angle hysteresis because the low hysteresis ensures easy rolling off of the water droplets, favourable for self-cleaning. Therefore, the contact angle hysteresis is a vital parameter for the characterization of the super-antiwetting surface. The $\theta_{\mathrm{Adv}}$ and $\theta_{\mathrm{Rec}}$ are typically measured using a commercial instrument with a professional

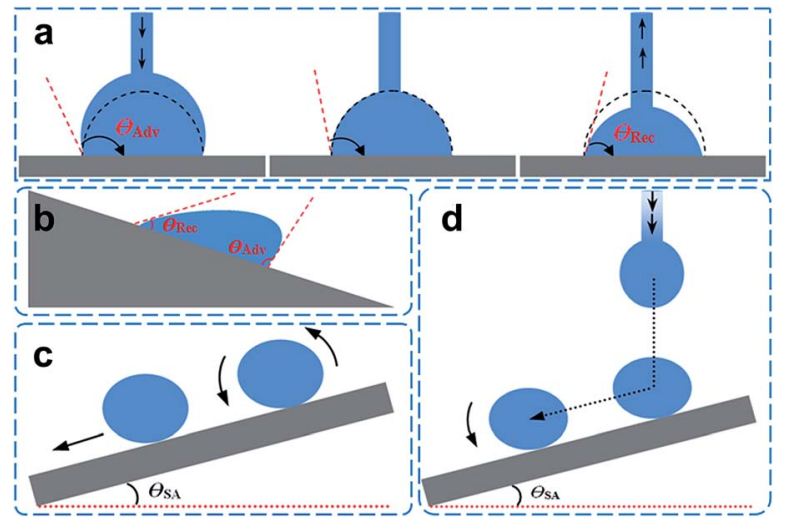

Fig. 3 Schematic illustrations of the technologies for characterization of the contact angle hysteresis: the advancing angle $\Theta_{\mathrm{Adv}}$ and the receding angle $\Theta_{\operatorname{Rec}}(a$ and $b)$, sliding angle or rolling angle $\Theta_{S A}$ (c and d).

and enhanced video microscope system with digital image analysis tools.

Two technologies are often used to measure the contact angle hysteresis, one is the dosing/decreasing method and the other is the tilting plate method (Fig. 3a and b). Some significant differences in the results have been reported between the two methods..$^{29-32}$ For example, the advancing angle and receding angles were measured in a Krüss DSA 100 (Germany) optical contact angle instrument. A syringe pump is used to generate a water droplet with controllable amounts. The rate of water pumping through the needle can also be controlled. After generating a water droplet, the volume of water droplet is further increased by further pumping water at a very low speed through the needle. At the beginning, the contact area between the water droplet and solid substrate does not change but the contact angle increases with increasing water droplet volume. When the water volume increases to a critical value, the three phase (liquid, solid, and air) contact line begins to move forward. We define the contact angle before the contact line change as the advancing angle. Similarly, when sucking the water back into the needle at a slow rate, the contact angle is prone to decrease with the decreasing liquid volume. The value of the receding angle is recorded at the point when the contact area begins to change. The final $\theta_{\mathrm{Adv}}$ and $\theta_{\mathrm{Rec}}$ are recorded simultaneously by a frame grabber using a charge coupled camera. Another method is by using a tilting plate, the tilt angle refers to the critical angle between the substrate and the horizontal surface, above which the droplet starts to move. It should be pointed out that the tilt angle reflects, but is not equal to the difference between $\theta_{\text {Adv }}$ and $\theta_{\text {Rec }}{ }^{33}$

\subsection{Sliding angle}

The sliding angle is empirical data to characterize a specific wettability surface. A low sliding angle is crucial to self-cleaning application. The sliding angle, also known as "roll-off" angle, is the one of the inclinations when the water droplet completely rolls off the surface without an external force. In the experiment, the contact angle measurement instrument is equipped 
with a tilting plate with a tunable angle from $0^{\circ}$ to $360^{\circ}$. The tilt angle is increased continuously from $0^{\circ}$ to $90^{\circ}$, and the angle is recorded as $\theta_{\mathrm{SA}}$ when the droplet is sliding away or rolling off the surface. ${ }^{34-36}$ There are mainly two methods to measure the sliding or roll-off angle based on the original state of water droplet contact with the substrate. Typically, a certain volume of water droplets is placed on a solid surface and the tilting plate then begins to tilt at a constant speed (Fig. 3c). When the water droplet slides or rolls off the surface, the tilt angle is regarded as the sliding angle. In most cases, researchers choose this method to obtain the sliding angle and it is convenient for macroscopically flat surfaces. For macroscopically rough surfaces, another technique is adopted, where a water droplet of defined volume is released onto the substrate with a certain height. The critical angle of inclination at which the substrate needs to be tilted until the droplet rolls off the surface is defined as the sliding angle (Fig. 3d). Several parameters need to be controlled and recorded in this case: the tilt angle of the substrate, the distance between the needle and substrate, the relative distance of the impact point to the lower end of the substrate, and the liquid volume.

\subsection{Adhesive force}

Normally, adhesive force is a direct index to evaluate the liquidsolid adhesion. Compared with the common static contact angle and dynamic sliding angle, there are much less literature studies reporting the adhesive force on the superhydrophobic surface. For the self-cleaning surface such as lotus leaves, well recognized as the Cassie state, water droplets cannot easily rest stable on the surface, demonstrating an ultralow adhesion force between the liquid and the substrate. However, an opposite example is the partially wetted "gecko" feet, which have extremely strong adhesive force that allows it to walk up and down on a vertical wall. Generally, a low adhesion force corresponds to a low sliding angle. The adhesion force of a superhydrophobic surface with a high static contact angle and a low sliding angle should be very small. Reversely, a surface with a lower adhesive force between the water droplet and substrate must exhibit excellent superhydrophobicity. The adhesive force is measured using a high-sensitivity micro-electrochemical balance system (Dataphysics DCAT11, Germany). A metal ring containing a certain volume of water droplets is placed above the sample and the sample table approaches, contacts and finally leaves the metal ring with a constant speed. The maximum force value (break peak point) measured during the sample table leaving process is taken as the value of adhesive force.

\section{Fabrication technologies of superhydrophobic textiles}

As discussed above, there are two significant factors in fabricating a superhydrophobic surface: the appropriate hierarchical structure with durable micro/nanoparticles and a low energy surface. For textiles with a micro-scale fibre structure, a common strategy is to coat nanoscale particles onto the fibre surface to achieve the micro/nanoscale structure and subsequently post-fluorinate the hierarchical structure for the low energy. The most common methods for preparing robust superhydrophobic textile surfaces include physical and chemical approaches, such as dip-coating, ${ }^{37-45}$ wet chemical deposition, ${ }^{\mathbf{4 6 - 4 9}}$ electro-assisted chemical deposition, ${ }^{50-54}$ spraycoating, ${ }^{55-57}$ sol-gel ${ }^{58-62}$ chemical etching, ${ }^{63-66}$ chemical vapour deposition, ${ }^{67-70}$ plasma processing, ${ }^{71-74}$ and polymer grafting. ${ }^{75-80}$ These available fabrication technologies will be separately discussed in the following categories: (i) pre-roughening and post-fluorinating, and (ii) simultaneous roughening and fluorinating (one-pot method).

\subsection{Pre-roughening and post-fluorinating}

The pre-roughening and post-fluorinating technology is the most common method available to date in preparation of cellulose-based superhydrophobic textile surfaces. Functionalization with nanoparticles or nanofilaments or a layer of film can usually achieve the required roughness. Nanoparticles such as $\mathrm{SiO}_{2}, \mathrm{TiO}_{2}$, and $\mathrm{ZnO}$ are often used to decorate textiles' surfaces to generate a roughness and durable superhydrophobic surface. In addition, some inorganic or organic chemical materials in the form of nanofilaments, nanofibres and even film layers are also reported in the literature to fabricate superhydrophobic cellulose-based surfaces. In this section, we will discuss in detail the fabrication technologies based on the formation type (particles, nanofilaments, nanofibre, and film) on the textile surfaces.

3.1.1 Dip-coating method. Wang et al. ${ }^{37}$ fabricated a superamphiphobic fabric with a significant self-healing effect against both physical and chemical damages via a two-step wet chemical route. As shown in Fig. 4, the cleaned fabric is immersed in a fluoroalkyl surface-modified silica nanoparticle solution for 5 minutes and then coated in fluorinated decyl polyhedral oligomeric silsesquioxane (FD-POSS) and fluoroalkylsilane (FAS) solution. The surface of polyester fabric exhibits a distinct protrusion structure after coating while the
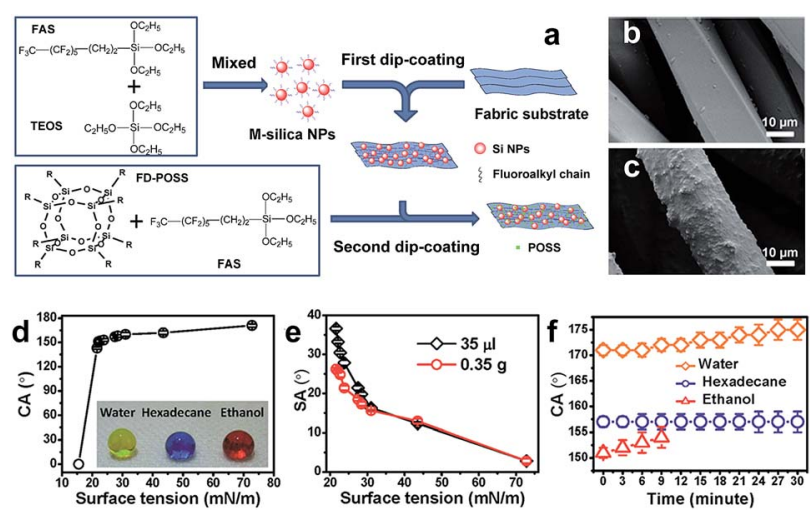

Fig. 4 (a) Chemical structures of coating materials and procedure for coating treatment. SEM images of (b) uncoated and (c) coated polyester fibres. The relationship of the CA (d) and SA (e) with surface tension and the CA changes of water, hexadecane and ethanol with time (f). (Reprinted from ref. 37 with permission). 
pristine polyester fabric surface is smooth. After coating, the fabric surface showed considerable liquid repellence to liquids with various surface tension and the CA remained above $150^{\circ}$ when the tested liquid has a surface tension greater than $22.1 \mathrm{mN} \mathrm{m}^{-1}$ (Fig. 4d). The dependency of the SA under constant liquid volume $(35 \mu \mathrm{L})$ and constant liquid weight $(0.35 \mathrm{~g})$ over different surface tension was also investigated, and the results indicated that the SA decreased with the increase of liquid surface tension (Fig. 4e). Moreover, the excellent wettability was confirmed by observing the contact angle changes on an extended period of time (Fig. 4f). In addition, the coated fabric indicates remarkable liquid repellence to water and low surface energy liquids including ethanol. After 200 cycles of the standard machine laundering process, the static contact angles did not obviously change. This suggests that the coating is durable enough to withstand the physical and chemical actions.

Similarly, $\mathrm{Xu}$ et $a .^{38}$ successfully prepared an enhanced stability superamphiphobic cotton fabric. In particular, a piece of cotton fabric is immersed in a chitosan aqueous solution to anchor amino groups onto cotton fibre. The sample was then deposited with silica nanoparticles. A robust structure was achieved by alternately depositing ormosil clusters onto a chitosan pre-coated cotton surface. Finally, the superamphiphobic cotton fabric was constructed by modifying with $1 \mathrm{H}, 1 \mathrm{H}, 2 \mathrm{H}, 2 \mathrm{H}$ perfluorodecyltriethoxysilane (PFDTES) to lower the surface energy. As predicted, the hierarchical structure cotton cellulose surface modification with low surface energy displays excellent anti-wetting properties. The superamphiphobic cotton fabric shows high contact angles of $164.4^{\circ}, 160.1^{\circ}$, and $156.3^{\circ}$ corresponding to water, cooking oil and hexadecane, and the water droplet and cooking oil can easily roll off from the cotton surface due to the extremely low adhesion of the cotton surface coating (Fig. 5b).

Liu et al. $^{42}$ reported a novel, highly effective coating strategy using a diblock copolymer consisting of a sol-gel forming polymer and a fluorinated block endowing the textiles with high water and oil repellence (Fig. 6). Cotton fabric was immersed into the diblock copolymer solution for $1 \mathrm{~min}$. Residue solvents were evaporated with a heating gun and subsequently the samples were annealed at a higher temperature. The water droplet, pump and cooking oils on such a superamphiphobic cotton surface can maintain their beaded shapes for months without being absorbed. Meanwhile, the obtained superamphiphobic property did

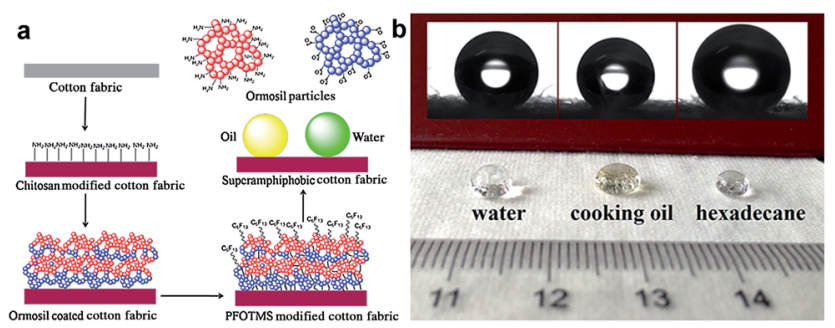

Fig. 5 (a) Schematic diagram of the fabrication process for superamphiphobic cotton fabrics. (b) Photographs of water, cooking oil and hexadecane droplets on the coated cotton fabrics. (Reprinted from ref. 38 with permission).

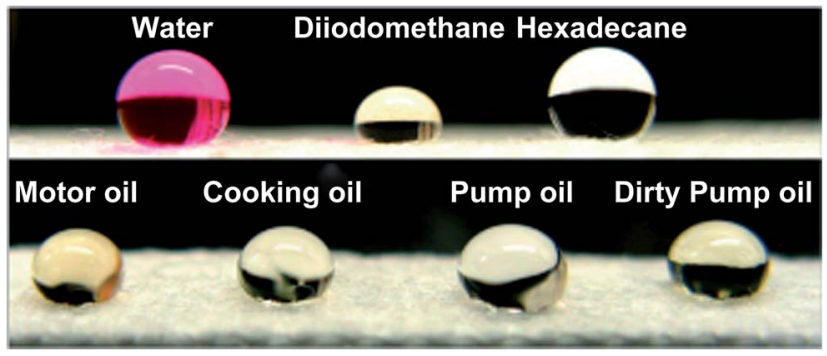

Fig. 6 Water droplet (dyed with rhodamine B) and various liquids on a cotton fabric grafted polymer with a concentration of $3 \mathrm{mg} \mathrm{mL}^{-1}$. (Reprinted from ref. 42 with permission).

not affect the mechanical stability and the coating was robust enough against simulated washing in detergent solutions. Moreover, they have synthesized other new diblock copolymers by sequential anionic polymerization and coated on various substrates, ${ }^{\mathbf{4 3 - 4 5}}$ such as paper, iron plates, glass plates and cell phone touchscreens.

3.1.2 Chemical bath deposition. Wang et $a .^{46}$ prepared a one-way transport oil droplet functional fabric surface by a two-step coating process to apply flower-like $\mathrm{ZnO}$ nanorods, fluorinated decyl polyhedral oligomeric silsesquioxanes, and hydrolyzed fluorinated alkylsilane. After the superamphiphobic fabric was exposed to UV light on one side, the fabric showed an interesting one-way oil transport ability. In addition, it was found that the selective oil-transportation depends on the specific surface tension of the liquid and by changing the UV irradiation time, different types of oils can be selected. Such

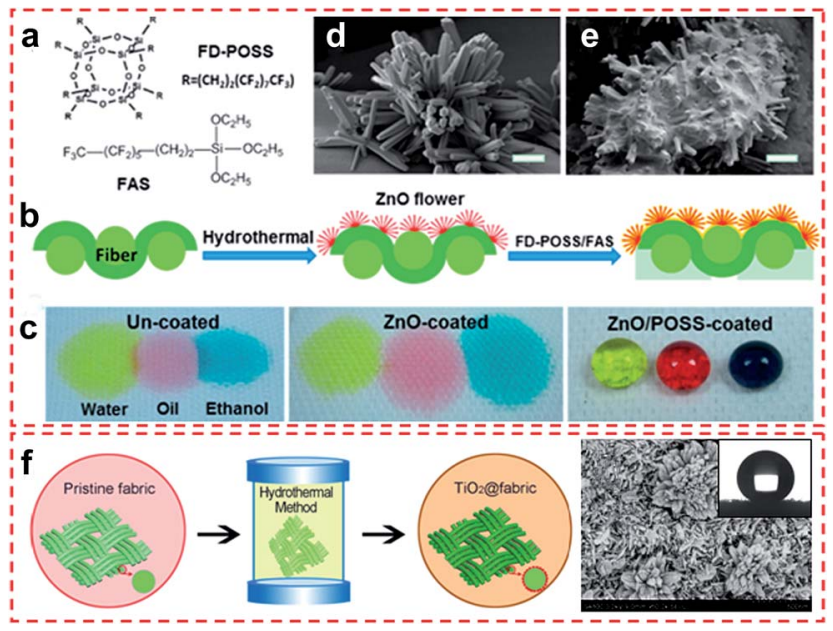

Fig. 7 (a) Chemical structures of the coating materials, (b) coating procedure, and (c) photographs of yellow-coloured water, red-coloured cooking oil, and blue-coloured ethanol (volume $35 \mu \mathrm{L}$ ) on cotton fabrics. SEM images of the cotton fabric (d) after $\mathrm{ZnO}$ treatment and (e) after both $\mathrm{ZnO}$ and FD-POSS/FAS treatment (scale bar $=2 \mu \mathrm{m}$ ). (Reprinted from ref. 46 with permission). (f) Schematic diagram of a facile one-pot hydrothermal process to construct $\mathrm{TiO}_{2}$ particle coating on fabrics. (g) High magnification SEM image of flower-like superhydrophobic $\mathrm{TiO}_{2}$ @Cotton fabricated at $150{ }^{\circ} \mathrm{C}$ for $20 \mathrm{~h}$; the inset is a photograph of a water droplet coated on the superhydrophobic surface. (Reprinted from ref. 47 with permission). 
a one-way oil fluid transport material has potential application in detecting liquid surface tension (Fig. 7a-e). In Lai and coworkers' work, ${ }^{47}$ a robust flower-like hierarchically structured cotton fabric surface was prepared in situ via one-pot hydrothermal technology, in which the cotton piece is immersed into
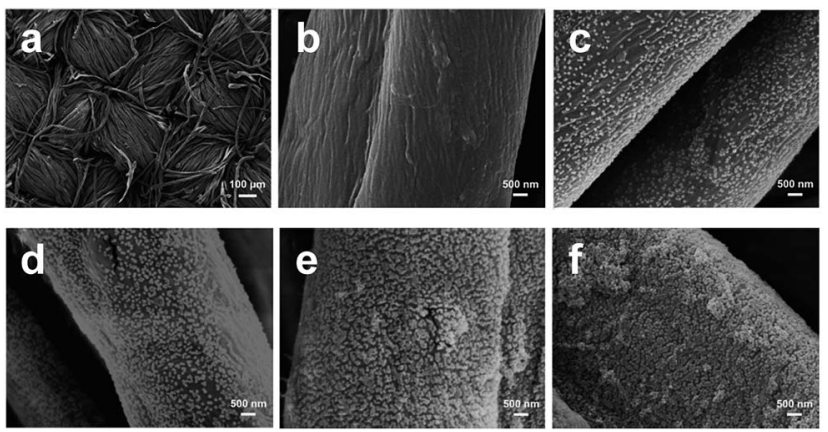

Fig. 8 SEM images of untreated cotton fabric ( $a$ and $b$ ) and cotton fabrics assembled with ( $\left.\mathrm{PAH} / \mathrm{SiO}_{2}\right)_{n}$ multilayers: (c) $n=1$, (d) $n=3$, (e) $n$ $=5$, and (f) $n=7$. (Reprinted from ref. 54 with permission). a solution mixture and treated at various temperatures ranging from $120{ }^{\circ} \mathrm{C}$ to $200{ }^{\circ} \mathrm{C}$ (Fig. 7f). Subsequently the remarkable superhydrophobic cotton surface was developed with the chemical modification of fluoroalkylsilane. The flower-like hierarchical $\mathrm{TiO}_{2}$ micro-nanoparticles were evenly coated on the cellulose fibre surface. Such special wetting superhydrophobic coating shows excellent water-repellent ability with a static contact angle larger than $160^{\circ}$ and a dynamic sliding angle less than $5^{\circ}$. The facile strategy for fabricating hierarchical structure superhydrophobic $\mathrm{TiO}_{2}$ @Cotton is excepted to be helpful for designing and developing self-cleaning materials in air and underwater superhydrophobic/superoleophilic materials.

3.1.3 Electrostatic layer by layer self-assembly. Lin et al. successfully prepared a superhydrophobic fabric surface via a versatile electrostatic layer by layer self-assembly method to construct polyelectrolyte/silica nanoparticle multilayers and a post-fluorinating strategy. ${ }^{54}$ Before the electrostatic selfassembly, the pristine cotton fabric was treated with specific solution to form a charged polymer film. Moreover, the surface morphology and hydrophobicity of cotton fabric were tuned by
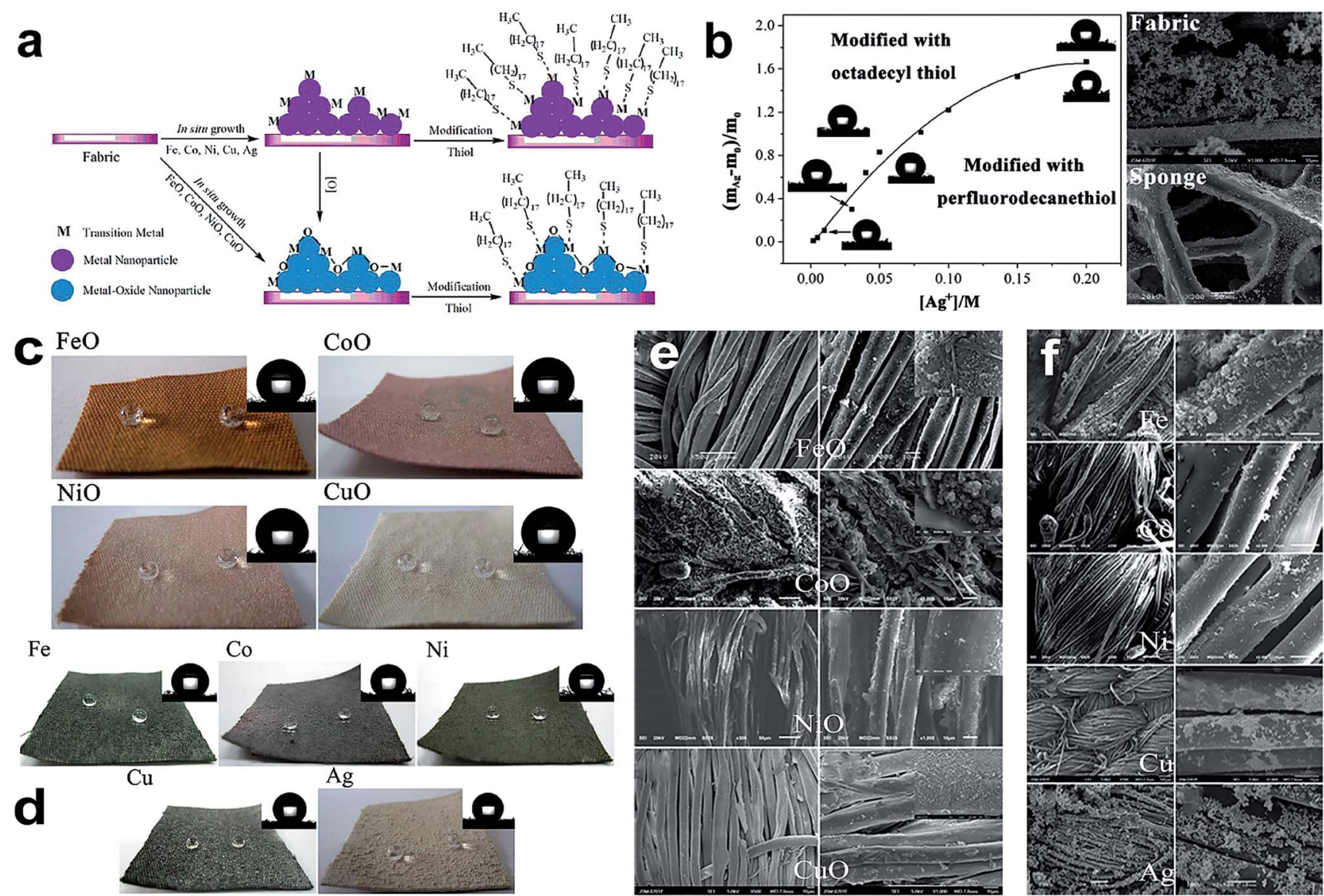

Fig. 9 (a) Schematic illustration of the preparation procedure of the superhydrophobic and superoleophilic fabric from the in situ growth of transition-metal/metal oxide nanocrystals with thiol modification. (b) Relationship between the proportion of weight increase $\left(\left(m_{\mathrm{Ag}}-m_{0}\right) / m_{0}\right)$ and the $\mathrm{Ag}^{+}$concentration. The images of the contact angle (CA) modified with both $n$-octadecyl thiol (upper left) and perfluorodecanethiol (lower right) at different concentrations are presented near the curve, and SEM images of fabric and sponge coated with Ag nanoparticles. Optical images of superhydrophobic fabric formed via the in situ growth of Group VIII and IB metal-oxide nanoparticles (c) and metal nanoparticles (d). The inset images in the upper right-hand corner of each panel are images of the static water droplets $(5 \mu \mathrm{L})$. SEM images of fabrics coated with transition-metal oxides (e) and metal (f) nanoparticles after surface modification with $n$-octadecyl thiol. (Reprinted from ref. 81 with permission). 
the number of silica-nanoparticle multilayers. The fabric exhibited a weave structure and the pure cotton fibre was very smooth (Fig. 8a and b). When the surface was assembled with 1 or 3 layers of $\mathrm{PHA}_{-} \mathrm{SiO}_{2}$, the silica-nanoparticles were dispersed onto the fibre surface randomly (Fig. 8c and d) and such a surface showed a sticky property with a high contact angle hysteresis. An increasing number of assemble layers could result in the homogeneous coverage and aggregation of silica nano-particles coated on the cotton fibre surface (Fig. 8e-h). The surfaces exhibited excellent superhydrophobicity with high static contact angles and low contact angle hysteresis. Meanwhile, the superhydrophobic surface can withstand at least 30 cycles of machine washing due to the good affinity between the $\mathrm{PHA}_{-} \mathrm{SiO}_{2}$ and cotton fibre.

3.1.4 In situ growth processing. Wang et al. ${ }^{81}$ reported a general methodology for in situ growth of transition metals and their oxide nanoparticles on fabric and sponge to realize stable surface roughness, which can readily coordinate with thiol, resulting in a special wetting property. It was also demonstrated that multi-scale surface roughness and the special wettability can be controlled via efficient control of the growth of nucleation of Group VIII and IB nanocrystals. The Group VIII and IB metal oxides, as well as simple metallic substances, such as $\mathrm{Fe}, \mathrm{Co}, \mathrm{Ni}$, $\mathrm{Cu}$, and $\mathrm{Ag}$ could provide the fabric/sponge different colours (Fig. 9c and d). The as-selected transition-metal element can not only strongly bond with thiols, but also possess some special properties that can be utilized to realize multifunctional integration. The multifunctional applications were mainly derived from magnetic recycling, semiconducting, and antibacterial material properties. Moreover, the as-prepared fabric and sponge via the in situ growth method followed by thiol modification possess anti-wettability towards water and can absorb and filtrate oils from water selectively with good efficiency.

\subsection{One-pot method}

Research on superhydrophobic fabric has attracted more and more attention and the number of literature studies has increased drastically in recent years. Inspired by the natural phenomenon of the lotus leaf surface, superhydrophobic surfaces have been successfully obtained on various substrates (cotton fabric, PET, wool, nonwoven and so on). As discussed earlier, superhydrophobicity depends on not only the chemical modification with low surface energy but also surface morphology to endow surface roughness. Besides the above discussed sequential roughening/lowering surface energy treatment, another strategy is by one-step dipping the samples into an appropriate pre-fluorinated micro-polymer or micro/ nanoparticle solution, or sol-gel solution. Other similar approaches include chemical coating, chemical vapour deposition, electrochemical deposition, layer by layer self-assembly and so on. Compared to the pre-roughing and subsequent postfluorinating technology, the one-pot method takes less time and is potentially cost-saving. Moreover, a one-step route can be more easily used in large scale application.

3.2.1 Wetting chemical coating. Lin et $a l .^{82}$ reported a robust, chemically stable superamphiphobic fabric prepared via a one-pot wetting coating method using a coating solution containing poly(vinylidene fluoride-cohexafluoropropylene) (PVDF-HFP), fluoroalkylsilane (FAS) and a volatile solvent (such as acetone) (Fig. 10a-c). The particle-free coating made the coated fabric super-repellent to liquids with a surface tension greater than $21.5 \mathrm{mN} \mathrm{m}^{-1}$ in acetone solution (Fig. 10d). Such a fabric surface showed good stability under continued liquid dropping with the static contact angle unchanged. It should be noted that the solution system of PVDF-HFP has a significant impact on superamphiphobicity and the threshold surface tension of coating to various liquids. For the fabric treated with DMF-acetone solution, the surface tension threshold is $30 \mathrm{mN}$ $\mathrm{m}^{-1}$ while the value changes to $32 \mathrm{mN} \mathrm{m}^{-1}$ when DMF was used as the solvent. By viewing the scanning electron microscopy image before and after the coating treatment, the pristine polyester fibre had a smooth surface while a rough morphology was formed on the treated polyester fibre no matter which solution system was adopted (Fig. 10e and f). Moreover, the coating surface exhibited high stability against the extreme conditions, such as $98 \% \mathrm{H}_{2} \mathrm{SO}_{4}, 40 \% \mathrm{NaOH}$, physical abrasion for 1000 cycles and machine wash for at least 800 cycles. The super-strong, superamphiphobic coating surface is useful for developing functional and innovative clothing surfaces.

3.2.2 Chemical vapor-phase deposition. Wang et al. ${ }^{83}$ reported a multifunctional fabric with electrical conductivity and superamphiphobicity by chemical vapour phase polymerization of 3,4-ethylenedioxythiophene (PEDOT) in the presence of $\mathrm{FeCl}_{3} \cdot 6 \mathrm{H}_{2} \mathrm{O}$, fluorinated decyl polyhedral oligomeric silsesquioxane (FD-POSS) and a fluorinated alkyl silane (FAS). The chemical structure and the chemical vapour phase polymerization
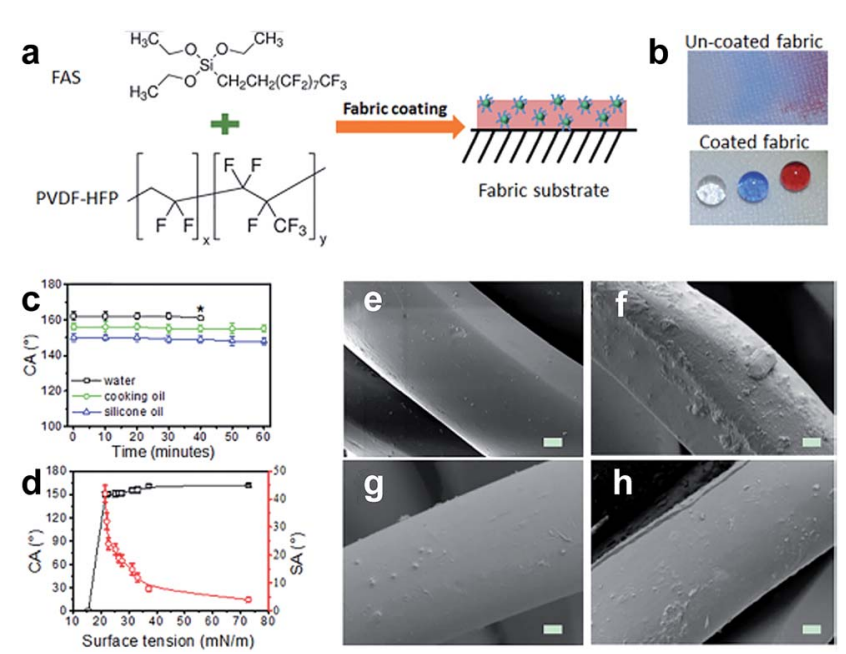

Fig. 10 (a) Chemical structures of coating materials and procedure for coating treatment, (b) water, olive oil, and silicone oil drops on the uncoated fabric and the coated fabric $(10 \mu \mathrm{L}$ each, and the dyes in the droplets showed no influence on the contact angles). (c) CA change with time (* is the point when the liquid drop evaporated over) and (d) dependency of the CA and SA on the surface tension of liquids. SEM images of polyester fibre (e) uncoated, ( $f$ ) coated in DMF-based coating solution, (g) coated in DMF/acetone ( $/ / v=1: 1)$ based coating solution, and (h) coated in acetone-based coating solution (scale bar = $2 \mu \mathrm{m}$, all coated fabrics were dried at $135{ }^{\circ} \mathrm{C}$ for $30 \mathrm{~min}$ ). (Reprinted from ref. 82 with permission). 
procedure are illustrated in Fig. 11a and b. The addition of FD-POSS and FAS endows the conductive coating with stable and durable superamphiphobicity with a contact angle of $169^{\circ}$ and $156^{\circ}$ to water and hexadecane, respectively; however the fabric surface with only PEDOT coating showed unstable hydrophobic properties and the water droplet will spread into the fabric matrix (Fig. 11e). In addition, the surface exhibited an excellent superamphiphobicity to liquids with surface tension larger than $27 \mathrm{mN}$ $\mathrm{m}^{-1}$ (Fig. 11g). FD-POSS and FAS were found to play an important role in enhancing the washing and abrasion durability and selfhealing function of the coating; meanwhile it showed little influence on the conductivity of coating. This facile and novel strategy will move forward the textile industry to durable and smart applications.

3.2.3 Polymer grafting. Textile materials, especially natural fibre such as cotton cellulose, silk and flax, exhibit excellent clothing properties due to their abundance, good moisture absorption, and biodegradability. However, the use of natural fibres in most advanced fields would be greatly restricted because of their poor chemical and physical properties. Atom transfer radical polymerization (ATRP) is a controlled radical polymerization technology that has been proven useful for synthesis of functional macromolecules with controlled architectures $^{\mathbf{8 4 - 8 8}}$ and the method has also been used for the modification of various substances. ${ }^{\mathbf{8 9 - 1 0 4}}$ In order to convert the hydrophilic cotton cellulose fibre into a superhydrophobic and self-cleaning surface, Nyström ${ }^{74}$ achieved it via surface in situ polymerization of glycidyl methacrylate using an ATRP method combined with a post-modification reaction. Based on the

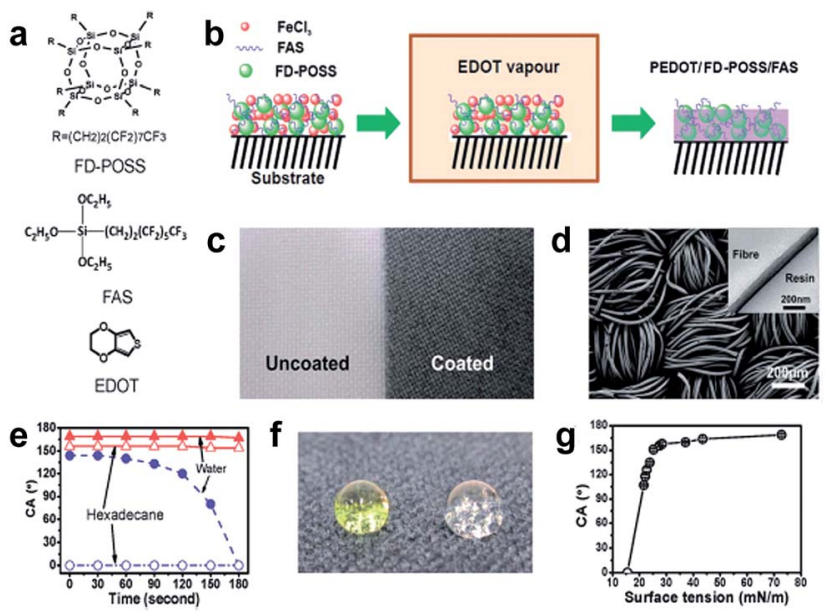

Fig. 11 (a) Chemical structures of FD-POSS, FAS and EDOT, (b) illustration of vapour-phase polymerization to form PEDOT/FD-POSS/FAS coating on fabrics, (c) photo of polyester fabric before (left) and after (right) coating treatment, (d) SEM image of the polyester fabric after coating with PEDOT/FD-POSS/FAS (inset is a cross-sectional TEM image of a PEDOT/FD-POSS/FAS coated fibre), (e) contact angle of the coated fabric changing over time from initial fluid-fabric contact, ( $f$ ) coloured water (yellow) and clear hexadecane drops on the PEDOT/ FD-POSS/FAS coated fabric (the surface tension of water and hexadecane is 72.80 and $27.47 \mathrm{mN} \mathrm{m}^{-1}$, at $20^{\circ} \mathrm{C}$ ), and (g) dependency of the contact angle on the surface tension of fluids. (Reprinted from ref. 83 with permission). environmental concerns over fluorine-containing compounds and their high cost, the work has demonstrated a versatile route to fabricate superhydrophobic and self-cleaning surfaces using low amounts or even no fluorinated materials. Similarly, Li et al. ${ }^{75}$ developed a highly hydrophobic silk material by grafting acrylate fluoride monomers via the ATRP route. The water repellence of the silk surface is controlled by the amount of fluoride monomers and it has little effect on the intrinsic properties of silk fabric. The thermal stability of the silk after modifying with a fluoride polymer was better than that of the pristine silk. Generally, the hydroxyl group is used to produce active sites for reacting with an initiator. Natural fibres containing abundant hydroxyl groups can be readily used to graft functional monomers. Besides the hydroxyl group, an aminocontaining dye intermediate (2-chloro-4,6-diamino-1,3,5triazine, CDATA) was also used to make more active sites for surface in situ polymerization of poly(methacrylic acid) (PMAA) in $\mathrm{Wu}$ and co-workers' work. ${ }^{76}$ The as-prepared cotton-PMAA exhibited high $\mathrm{pH}$-responsibility: with increasing $\mathrm{pH}$, the surface wettability transited from superhydrophobic to superhydrophilic and underwater superoleophobic, leading to efficient water/oil separation.

Typically, a long fluoroalkyl chain polymer monomer is adopted to modify various substrates with excellent and stable superhydrophobicity. Taking account of the environmental protection and high cost of the long chain fluoride-based precursor, Lai et al. ${ }^{77}$ grafted a short chain fluorine-containing monomer on a cotton fabric surface by the ATRP strategy for the construction of super-antiwetting fabric with controllable surface adhesions, excellent air permeability, and good mechanical stability under dry abrasion and wet laundering processes. In this work, a single graft route was adopted to fabricate super-antiwetting surfaces with high surface adhesion (Fig. 12a). The robust and stable superhydrophobic surface was developed by employing a graft-on-graft route (Fig. 12b), and the as-prepared fabric surface indicated a high static water contact angle $\left(163.7 \pm 2.5^{\circ}\right)$ with lower adhesion (Fig. 12c). These results verify that the designed graft-on-graft architecture is an effective and promising approach to achieve excellent super-anti-wetting ability with environmentally friendly short fluoroalkyl chains. This chain grafting technique has realized the construction of superhydrophobic coatings in a sophisticated manner through closely controlled chemical reactions, but it has a drawback that it needs a specific multi-step process and is time-consuming. Xue et al. ${ }^{78}$ prepared a robust and antifouling superhydrophobic PET fabric surface via a novel and general strategy through surface initiated atom transmit radical polymerization of fluorinated methacrylates on poly(ethylene terephthalate) (PET) fabrics. Briefly, the cleaned PET fabric was firstly immersed in a specific concentration of sodium hydroxide solution for chemical etching to obtain abundant hydroxyl groups. Then $\alpha$ bromoisobutyryl bromide (BiBB) as the ATRP initiator was used to immobilize the $\mathrm{Br}$ group on the etched PET fabric surface (denoted as PET-Br). Finally the fluorinated methacrylate polymer grafted on the PET-Br and the resultant fabric was denoted as PET-g-P(TFEMA) (Fig. 12d). After grafting polymerization, the PET- $g$-P(TFEMA) fabric surface possessed water 

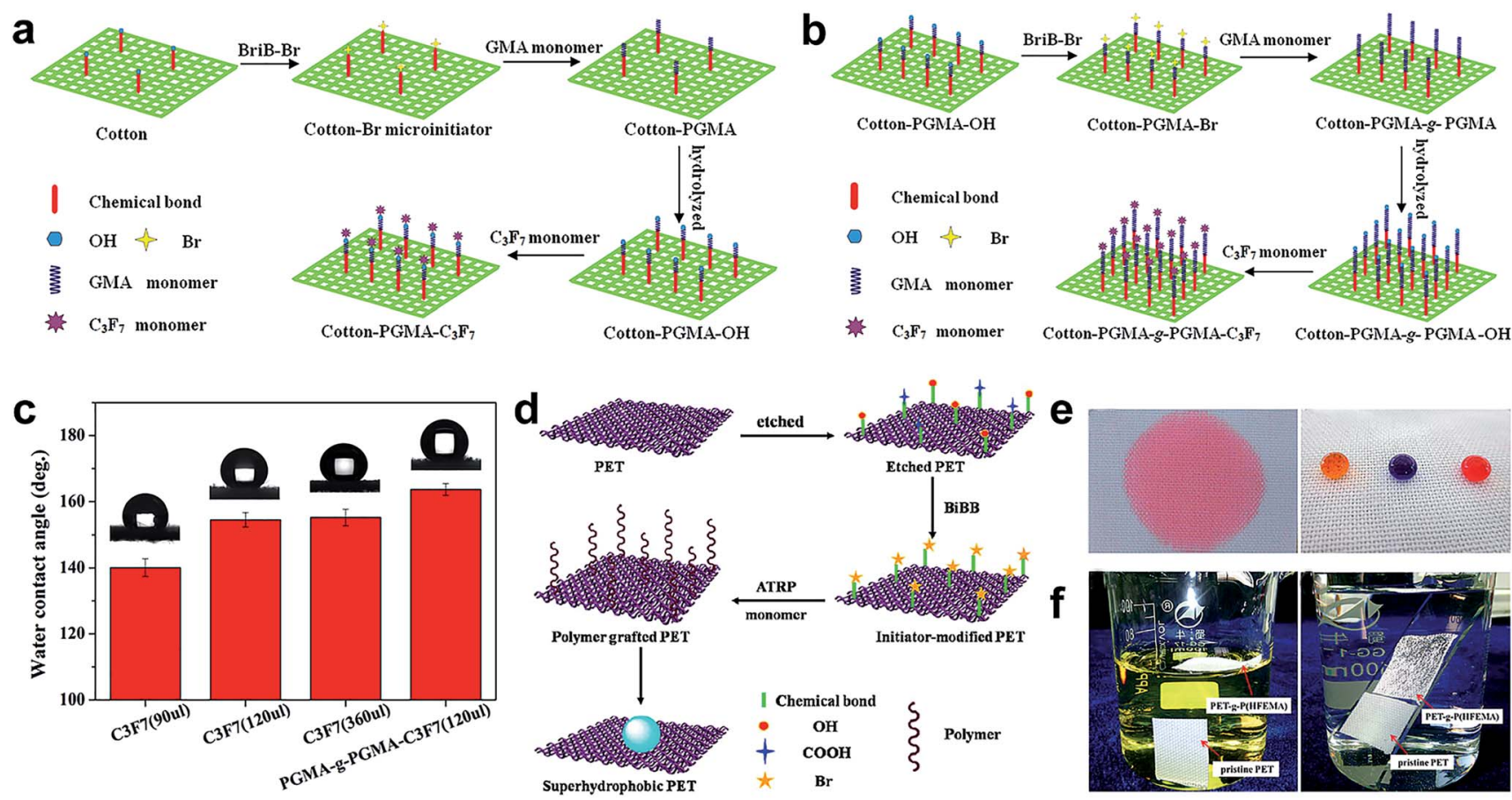

Fig. 12 Schematic diagrams of the synthetic superhydrophobic cotton surface by grafting the fluoroalkyl chain (a) and graft-on-graft route (b). The contact angle changes on grafted functional cotton samples with different fluoroalkyl amounts and graft-on-graft surface (c). (Reprinted from ref. 77 with permission). The schematic illustration of fabrication of the superhydrophobic PET fabric surface (d). Photographs of dyed water droplets on pristine and PET-g-P(TFEMA) fabric (e) and the wetting behaviour of pristine and PET-g-P(TFEMA) fabric (f). (Reprinted from ref. 78 with permission).

repellence while the pristine PET fabric is superhydrophilic (Fig. 12e). Meanwhile, the as-prepared superhydrophobic fabric float onto the water surface and a special "silver mirror" phenomenon was observed (Fig. 12f), indicating a remarkable super-anti-wettability. The hydrophobicity of the as-prepared PET surface can be tuned by altering the polymerization time. It was verified that the superhydrophobic surface was resistant to UV irradiation, chemical solutions such as acid, base, salt, acetone, and toluene, mechanical laundering, and mechanical abrasion. It is notable that such fabric remained superhydrophobic after 2500 mechanical laundering cycles, 100 abrasion cycles and even long time exposure to UV irradiation.

$\mathrm{Hu}$ et al. ${ }^{99-104}$ have also fabricated superhydrophobic textiles using fluorinated block polymers via atom transfer radical polymerization. In their research, a highly durable and robust superhydrophobic fabric surface was fabricated with a series of functional diblock copolymers. ${ }^{99}$ The nano/micro-scale roughness was synthesised in a single step by combining the copolymer-based nano-bumps onto the cotton fiber, while the poly(2,2,2-trifluoroethylmethacrylate) (PTFEMA) blocks acted as a low surface energy material. Since the low-fluorinated PTFEMA chains were chemically bonded with the cotton fiber, the prepared superamphiphobic cotton fabric surface exhibited super-high durability, long-term stability and robustness. Although many technologies have been used for the fabrication of superhydrophobic textiles, the common drawback in these strategies is the usage of organic solutions which can inhibit the practical applications. Hu et al. reported an aqueous process for preparing robust diblock copolymer coating superamphiphobic surfaces. ${ }^{100}$ The diblock copolymer of poly(2-perfluorooctylethyl acrylate)-block-poly(glycidyl methacrylate-radom-methoxy oligoethyleneglycolyl methacrylate) [PFOEA- $b$-P(GMA-r-mOEGMA)] was synthesised in water solution via atom transfer radical polymerization (Fig. 13a and b). Cotton fabric was soaked in the formed coating solution and can achieve robust wettability. In addition, the coating structure can be tuned by changing copolymer solution concentration. Fig. 13c-k compare the SEM and 3D AFM images of uncoated and coated PET cotton. The coated cotton at $c=2.28 \mathrm{mg} \mathrm{mL}^{-1}$ appeared rather smooth; however, the wettability against water and oil droplets was rather weaker compared to the coating at $c=22.8 \mathrm{mg} \mathrm{mL}^{-1}$, reaching a superamphiphobic level. Furthermore, they can withstand ten cycles of simulated laundry and harsh rubbing cycles. This novel process is simple, environmentally friendly and expected to find commercial applications.

\section{Multifunctional applications}

Nowadays, research studies on special wetting materials have become a hot spot in the development of advanced materials. ${ }^{105-107}$ With the increasing demands for multifunctional advanced materials with super-antiwetting properties, great attention and interest have triggered scientists and researchers to focus on special wetting surfaces for a large number of functional applications. Most prominently, special wetting surfaces are regarded as excellent oil/water separation materials 

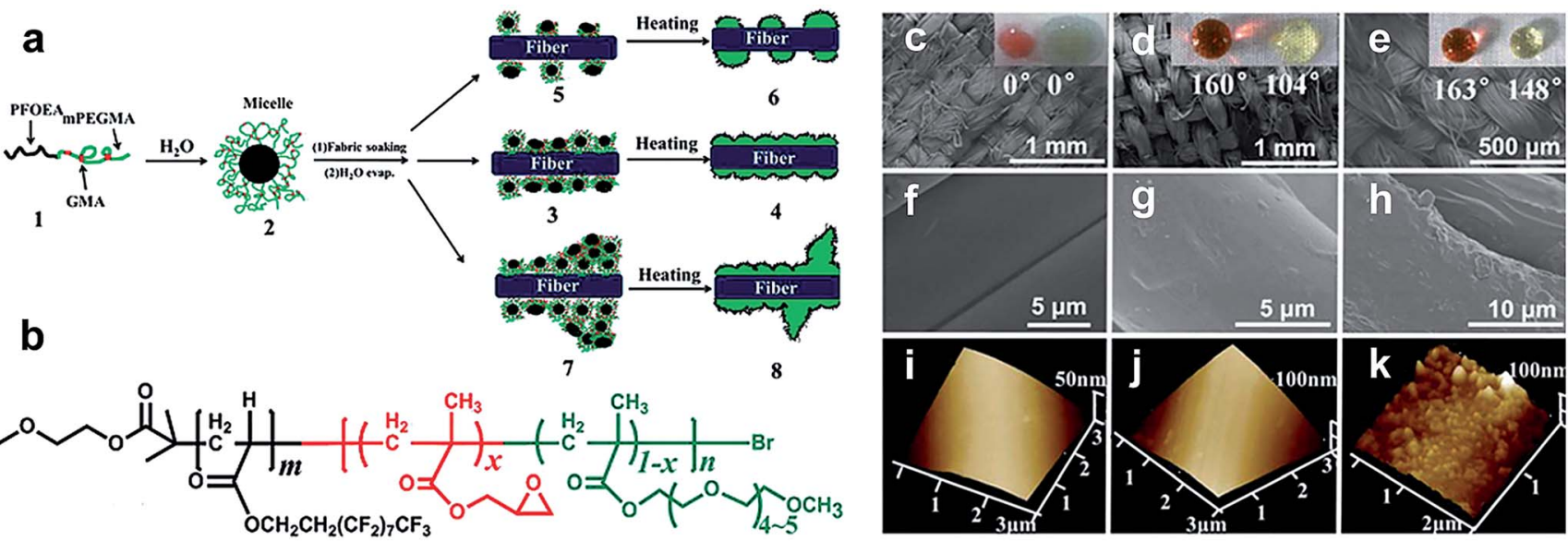

Fig. 13 (a) Schematic illustration of grafting a diblock copolymer on cotton fabric via atom transfer radical polymerization. (b) Chemical structure of the diblock copolymer: PFOEA-b-P(GMA-r-mOEGMA). SEM images (c-h) and 3D AFM images (i-k) of fabric yarns and fibers. (c, f and i) for uncoated cotton fabric, fabric coated at $c=2.28 \mathrm{mg} \mathrm{mL}^{-1}\left(\mathrm{~d}, \mathrm{~g}\right.$ and $\mathrm{j}$ ) and $22.8 \mathrm{mg} \mathrm{mL}^{-1}(\mathrm{e}, \mathrm{h}$ and $\mathrm{k}$ ), respectively. The insets (c-e) are optical images of the water droplet (dyed with red) and peanut cooking oil droplet sitting on fabric surfaces, respectively. (Reprinted from ref. 100 with permission).

due to the different wettability to water and oil, such as being superhydrophobic and superhydrophilic, superoleophilic and superoleophobic. Textiles with a textured structure are very easily attacked by various chemical solvents (acid, base, salt, organic and inorganic solution) and lose their intrinsic properties. Superhydrophobic textiles fabricated by constructing appropriate surface roughness and suitable chemistry have been successfully demonstrated for a range of applications, such as oil/water separation, self-cleaning, and multifunctional materials containing UV-shielding, flame-retardant, anti-icing, and photocatalytic properties, as well as some smart materials with self-healing, stimuli-responsive, patterning and asymmetric response, etc. In this section, we will mainly focus on the functional applications of textiles (mainly on cellulose based materials) with special wettability surfaces.

\subsection{Superamphiphobic surface}

Most applications of superamphiphobic surfaces are related to their versatile anti-wetting property. Being both superhydrophobic and superoleophobic, they have similar features and application potential as the superhydrophobic-only surface, but there are exceptions. For example, a superhydrophobic surface can be used as a filter to separate oil from a water mixture if its surface wetting states are properly tuned. Obviously, superamphiphobic surfaces cannot be applied in oil/ water separation because of their super-antiwetting to both types of liquids. Superamphiphobic surfaces should be developed for advanced textiles such as totally anti-wetting surface, anti-fouling, and packaging materials for precision instruments where anti-wettability is expected for not only pure water but also for some low surface energy solutions (e.g., detergent solution, rain water, underground water, waste water, and sea water) and organic 'oils' (e.g., hexane, hexadecane, toluene, mineral oil, and cooking oil). ${ }^{108}$

Artus et al. ${ }^{109}$ fabricated a superoleophobic polyester fabric by coating silicone nanofilaments, followed by plasma fluorination.
Such a fabric surface was revealed to have outstanding repellence against static liquid drops with low surface energy and exhibited a possible highest oil-repellence grade of eight. Meanwhile, Leng' groups ${ }^{110}$ reported a superoleophobic woven fabric with a multilength scale structure by adding two layers of silica particles (microparticles and nanoparticles) on the woven structure. Subsequently, the multi-length scale roughness fabric was incorporated with fluoroalkyl groups to obtain superoleophobic properties. As expected, the modified woven fabric demonstrated a high hexadecane static contact angle ( $153^{\circ}$ for $5 \mu \mathrm{L}$ droplets) and low roll-off angle ( $9^{\circ}$ for $20 \mu \mathrm{L}$ droplets). At the same time, the necessity to add the nanoparticle layer onto the fibre surface for preparing superoleophobicity was also proved, especially to achieve the low sliding angles of oil droplets.

Although any surface can be defined as superoleophobic as long as it has a contact angle greater than $150^{\circ}$, unlike the superhydrophobic state, the measured surface properties of superoleophobicity could be considerably different using oily fluids with different surface energy. In other words, a superoleophobic surface to a certain oily fluid may become oleophilic to some other oily fluids with lower surface tension. Understandably, making a super-repellent surface to oils with a high surface tension is easier than fabricating the same against a lower surface energy oil. The latter has always been a great challenge. With the development of superhydrophobic/superoleophobic technologies, stimuli-responsive superhydrophobic surfaces by an external stimulus such as thermal, electrical, magnetic field and light have been of particular interest in recent years. Wang et al. ${ }^{111}$ adopted one-pot vapour phase polymerization of pyrrole (PPy) onto a fluorinated alkyl silane (FAS) fabric leading to a superamphiphobic flexible polyester fabric surface. The PPy-FAS coated fabric has demonstrated a high contact angle of $165 \pm 2^{\circ}$ and $154 \pm 2^{\circ}$ to water and hexadecane oil, respectively. Furthermore, for the oily fluids of surface tension larger than $27 \mathrm{mN} \mathrm{m}^{-1}$, the PPy-FAS treated cotton fabric has a contact angle above $150^{\circ}$, showing an excellent super-repellent characteristic. Meanwhile, the coatings demonstrated durable 
laundering and robust mechanical stability, attributed to the superamphiphobicity. In Wang and co-workers' research, ${ }^{\mathbf{1 1 2}}$ a multifunctional super-repellent liquid fabric surface was successfully fabricated using hydrolysis of fluorinated-decyl polyhedral oligomeric silsesquioxane (FD-POSS) and a fluorinated alkyl silane (FAS). The coated fabric exhibited excellent self-healing performance after UV exposure, acid exposure, machine wash and mechanical abrasion.

In spite of the excellent super-repellent property of both water and oily fluids of cotton fabric developed by hydrolysis of FD-POSS and FAS, the commercial unavailability of FD-POSS imposes a restriction on the wide use of this coating technology for practical application. Zhou et al. ${ }^{\mathbf{1 1 3}}$ reported a new coating system to make a durable self-healing superamphiphobic fabric surface via a two-step dip coating method (Fig. 14b). They prepared two coating solutions, one consisting of nanoparticle silica sol and FAS in ethanol under alkaline conditions, and the other a fluoro-containing polymer, poly(vinylidene fluoridehexafluoropropylene) (PVDF-HFP) dispersed in dimethylformamide (DMF) solution containing FAS. The fabric was immersed into the solutions in sequence. The coated fabric showed super-antiwettability and can withstand at least 600 cycles of standard laundry (Fig. 14c and d) as well as 8000 cycles of mechanical abrasion (Fig. 14g and h) without any apparent change in superamphiphobic properties. Moreover, the coated fabric has self-healing ability with a short-time thermal treatment or long time ageing after exposure to extreme conditions, such as acid/base, ozone and boiling treatment. Interestingly, a new route for fabricating superamphiphobic fabric was also developed by a dip-coating method by $\mathrm{Xu}$ and co-workers. ${ }^{114} \mathrm{~A}$ solution containing heptadecafluorononanoic acid-modified $\mathrm{TiO}_{2}$-sol mixed with silica nanoparticles was used to apply onto the polyester fabric surface. The superamphiphobic coating was indicated to have a stable super-antiwetting water angle contact above $150^{\circ}$ in air; however, the superhydrophobic surface became superhydrophilic when exposed to ammonia vapour.

\subsection{Self-cleaning/photocatalytic/anti-bacterial}

Self-cleaning coatings can be broadly classified into two major types: photocatalysis-induced superhydrophilic coatings and superhydrophobic or superamphiphobic coatings. ${ }^{115}$ In terms of superamiphobic or superamphiphobic surfaces, an air layer gets trapped between the nano/microstructure and water droplet, resulting in the formation of a composite air/liquid/ solid interface and increase in the contact angle. A liquid droplet can easily roll off from the fabric surface and take away the surface dirt. For superhydrophilic coatings, the surface is cleaned by exerting the photocatalytic effect which breaks down the organic substances into carbon dioxide and water. The selfcleaning property of super-antiwetting fabric is summarized as three types: physical self-cleaning, chemical self-cleaning and biological self-cleaning. Physical self-cleaning is mainly mimicking the lotus leaf surface and characterized by measuring the water contact angle and the sliding angle. Chemical self-cleaning refers to the degradation of the colour stain or pollution solution using the photocatalytic effect. The biological self-cleaning corresponds to antibacterial activity of functional fabric against a Gram-positive bacterium (Staphyloccocus aureus) and a Gram-negative bacterium (Escherichia coli).

Typically, superamphiphobic or superhydrophobic surfaces with the water contact angle above $150^{\circ}$ and an ultralow sliding angle are endowed with physical self-cleaning properties. Lin et al. ${ }^{116}$ reported a self-cleaning cotton fabric surface coated with a superhydrophobic and superoleophobic thin composite polymer film consisting of modified $\mathrm{SiO}_{2}$ nanoparticles and a fluoropolymer. As shown in Fig. 15a, a water droplet and
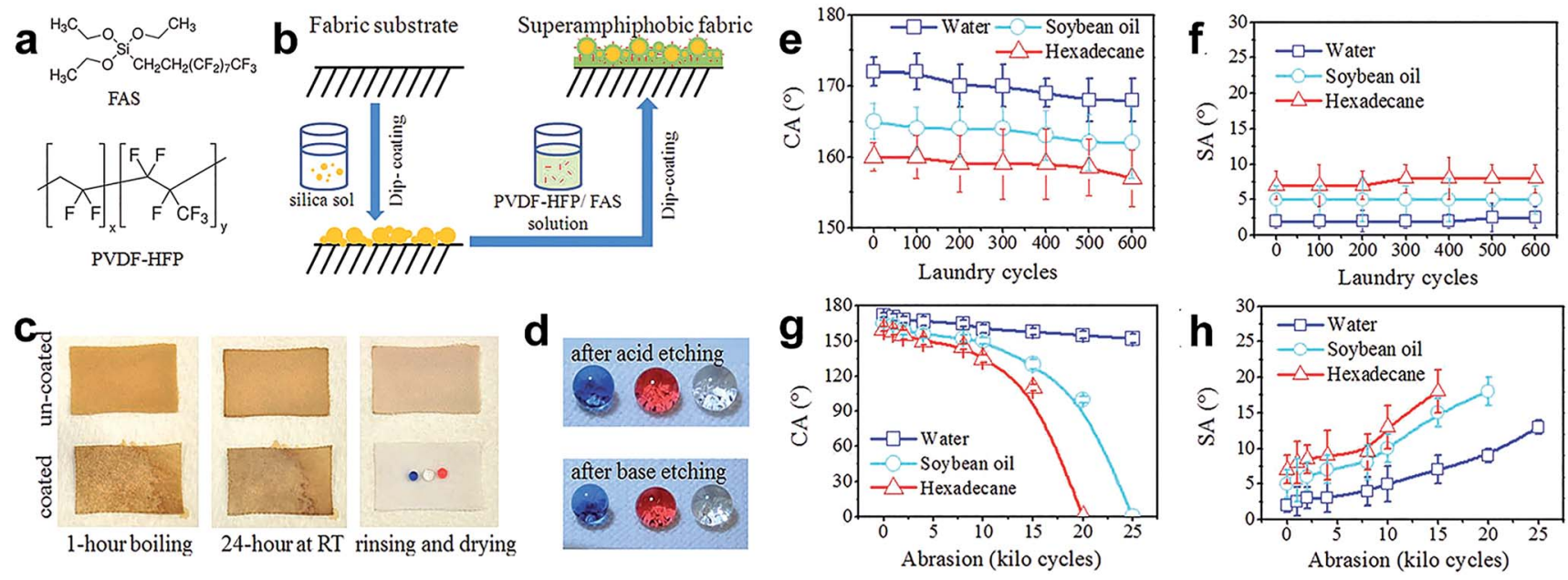

Fig. 14 (a) Chemical structure of FAS and PVDF-HFP. (b) Coating procedure for superamphiphobic fabrics. (c) CA and (d) SA change with laundry cycles. (e) Photographs of uncoated and coated polyester fabrics after being boiled in coffee, left at room temperature for $24 \mathrm{~h}$, rinsed with water, and then dried at room temperature. (f) Photographs of colored liquid droplets (blue water, red hexadecane and clear soybean oil, all in $10 \mu \mathrm{L}$ ) on the coated fabric after immersion in strong acid or base solution for 7 days (after acid etching $\mathrm{CA}: 170^{\circ}, 163^{\circ}$ and $160^{\circ}$ for water, soybean oil and hexadecane, $\mathrm{SA}: 2^{\circ}, 5^{\circ}$ and $8^{\circ}$ respectively; after base etching $\mathrm{CA}: 170^{\circ}, 164^{\circ}$ and $160^{\circ} ; \mathrm{SA}: 2.5^{\circ}, 5^{\circ}$ and $7.5^{\circ}$ for water, soybean and hexadecane, respectively). (g) CA and (h) SA change with abrasion cycles. (Reprinted from ref. 113 with permission). 

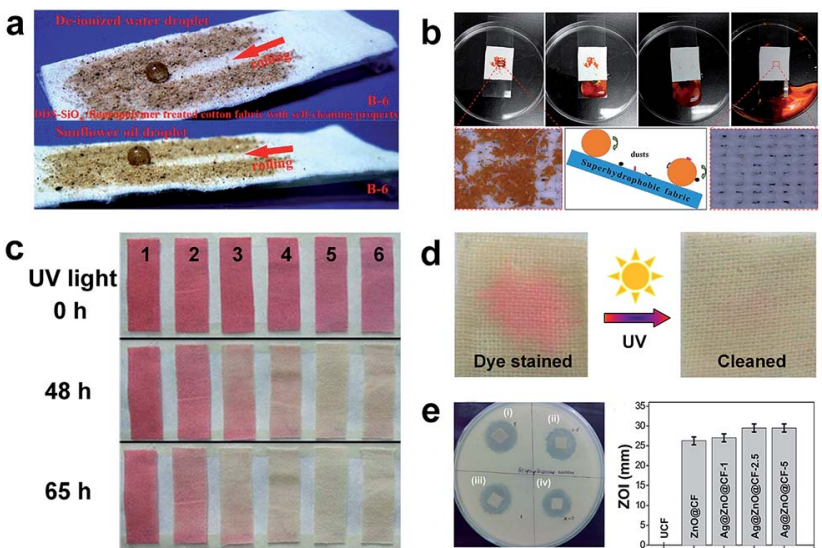

Fig. 15 (a) Self-cleaning property of superamphiphobic fabric prepared by coating $\mathrm{SiO}_{2}$ /fluoropolymer (reprinted from ref. 116 with permission); (b) self-cleaning behaviour of the superhydrophobic $\mathrm{TiO}_{2}$ (CCotton surface (reprinted from ref. 47 with permission); (c) photocatalyzed self-cleaning of various fabrics under UV irradiation $\left(355 \mathrm{~nm}, 2.0 \pm 0.1 \mathrm{~mW} \mathrm{~cm}^{-2}\right.$ ) for different times. The numbers inserted in (c) represent 1: cotton fabric, 2: cotton-g-PHEA (DGHEA: 6.9\%), 3: cotton- $g-\mathrm{TiO}_{2}$ (DG $\mathrm{HEA}: 5.9 \%, \mathrm{DG}_{\mathrm{TiO}_{2}}: 0.69 \%$ ), 4: cotton- $g-\mathrm{TiO}_{2}$ (DGHEA $: 9.6 \%, \mathrm{DG}_{\mathrm{TiO}_{2}}: 2.3 \%$ ), 5: cotton- $g-\mathrm{TiO}_{2}$ (DG $\mathrm{HEA}: 12.5 \%, \mathrm{DG}_{\mathrm{TiO}_{2}}$ : $5.2 \%$ ), and 6: cotton-g- $\mathrm{TiO}_{2}\left(\mathrm{DG}_{\mathrm{HEA}}: 17 \%, \mathrm{DG}_{\mathrm{TiO}_{2}}: 6.5 \%\right.$ ) (reprinted from ref. 119 with permission); (d) photocatalytic self-cleaning of $\mathrm{Ag} /$ $\mathrm{ZnO}$ aCF-2.5 coated fabric stained with RhB before and after exposure to sunlight for $15 \mathrm{~h}$. (e) Biological self-cleaning of Ag/ZnO@CF-2.5 coated fabric by measuring the inhibition zone. (Reprinted from ref. 107 with permission).

a sunflower oil rolled off from the as-prepared cotton fabric surface and brought away dirt along with it. A clear track was left behind by a spherical water droplet or oil droplet. Similarly, in Lai's group, ${ }^{47}$ flower-like $\mathrm{TiO}_{2}$ micro/nanoparticles coated on the cotton fabric surface and subsequently modified with fluoroalkylsilane. The superhydrophobic cotton surface exhibited a super-antiwetting property with self-cleaning ability and oil/ water separation (Fig. 15b). A literature study in Science reported a facile method to fabricate superamphiphobic selfcleaning surfaces on both hard and soft materials which were obtained by preparing a suspension of perfluorosilane $\mathrm{TiO}_{2}$ nanoparticles and bonding with commercial adhesives. These surfaces were self-cleaning even after immersion in oils and they could maintain super-repellence after 40 cycles of abrasions under sandpaper. ${ }^{117}$

It is well known that the lotus-leaf surface exhibits selfcleaning properties due to the superhydrophobicity which can remove dust away from the surface. However, the physical selfcleaning process is unable to clean oil pollutants if the surface is superhydrophobic and oleophilic. Thus, the incorporation of $\mathrm{ZnO}$ or $\mathrm{TiO}_{2}$ nanoparticles becomes indispensable to degrade organics by the photocatalytic effect. Zhang et al. ${ }^{\mathbf{1 1 8}}$ developed a photocatalysis-induced self-cleaning polyester non-woven fabric surface by dip-coating a fluorine-free low surface energy polybenzoxazine (PBZ) solution with $\mathrm{TiO}_{2}$ nanoparticles, followed by thermal treatment. The oil droplet on the treated fabric surface can be photodegraded by $\mathrm{TiO}_{2}$ endowing the fabric with self-cleaning performance. Yu et al. ${ }^{119}$ used a new strategy to covalently immobilize $\mathrm{TiO}_{2}$ nanoparticles onto the fabric surface by grafting polymerization of 2-hydroxyethyl acrylate (HEA) under $\gamma$-ray irradiation. The resulting functional fabric showed photocatalytic self-cleaning performance when using oleic acid dyed with oil red as the organic stain (Fig. 15c). As a comparison, the colours of pristine cotton were always red no matter how long it was exposed to the UV irradiation. Reversely, the red colours of various cotton- $g-\mathrm{TiO}_{2}$ gradually disappeared under ultraviolet irradiation because of the photocatalytic effect of the $\mathrm{TiO}_{2}$ nanoparticles on the surface. Rana et al. $^{\mathbf{1 2 0}}$ adopted a biomimetic method to construct nanostructured Ag@ZnO on the cotton fabric surface; such flexible fabrics exhibited not only visible-light driven photocatalysis but also anti-bacterial activity against Gram-positive bacteria and Gram-negative bacteria (Fig. 15d).

\subsection{Oil/water separation}

The increase of oil leakage and oil spill accidents pose a great threat to the aquatic environment. A great amount of attention has been focused on oil spill prevention and clean-up; special wetting materials are widely applied to separate the oil/water mixture due to the opposing affinities toward water and oil. In particular, two kinds of wettability materials are suitable for oil/ water separation: hydrophobic/oleophilic and hydrophilic/ oleophobic. In principle, the wetting behaviour is characterized by the surface chemistry and can be boosted by the surface structure. ${ }^{\mathbf{1 2 1 , 1 2 2}}$ With a suitable surface roughness, the wettability can be tuned from hydrophobic to superhydrophobic, oleophilic to superoleophilic. Indeed, as textured textile materials are flexible, they are suitable for absorbing organic solutions and separating the oil/water mixture, once the appropriate synergistic effect between the surface chemistry material and surface architecture is employed. ${ }^{123-145}$ So far, based on the principle of separating two different surface tension solution mixtures, the special wettability textile can be divided into two types: as a filtration membrane and as an absorption material. The filtration membrane allows only oil or water to permeate through and repels the other phase, resulting in a selective separation. The absorption material can selectively absorb water or oil on the surface, and thus prevent the other phase from permeating into the absorbent.

Rana et al. ${ }^{129}$ reported a multifunctional cotton fabric with $\mathrm{Ag} / \mathrm{AgBr}-\mathrm{TiO}_{2}$ composite nanoparticles and silane coating via a simple spray-coating method. The resulting cotton fabric showed an enhanced mechanical property in both warp and weft directions. Moreover, the developed fabric also exhibited selective absorption of oil from the oil/water mixture. Zhou et $a{ }^{130}$ used a simple and versatile approach to fabricate superhydrophobic and superoleophilic surfaces by introducing polyaniline and fluorinated alkyl silane onto cotton fabric. The as-prepared cotton surface can be regarded as a separation material for the oil/water mixture with a high separation efficiency of $97.8 \%$. The separation process can be repeated for at least 30 cycles without an apparent decrease of separation efficiency. Besides, the obtained fabric can also withstand extreme conditions, such as high temperature, high humidity, strong 
acid and alkaline conditions, and even the mechanical abrasion. Based on the above report, a facile and inexpensively onepot sonochemistry irradiation method was developed for constructing two-side superhydrophobic fabric incorporated with $\mathrm{SiO}_{2}$ nanoparticles (Fig. 16a). ${ }^{132}$ The resulting fabric exhibited both superhydrophobicity and superoleophilicity with a high water contact angle of $159^{\circ}$ and oil contact angle of nearly $0^{\circ}$, which is suitable for oil/water separation. The fabric can be used for capturing and separating various oils both on the water surface and underwater, including toluene, chloroform, kerosene, etc. (Fig. 16b). Furthermore, a superhydrophobic contact angle above $150^{\circ}$ and excellent separation efficiency beyond $94.6 \%$ were observed after 40 separation cycles. In addition, the obtained fabric showed stable and robust superhydrophobicity against hot water, strong acid, alkaline, salt solution and mechanical abrasion. This stable and durable superhydrophobic fabric surface has the greatest potential for practical applications. Chen et al. ${ }^{133}$ reported a simple and practical route to develop a superhydrophobic $\mathrm{SiO}_{2}-\mathrm{TiO}_{2} @$ PDMS hybrid film via a sol-gel method, which endows the coated polyester-cotton fabric surface with superhydrophobicity and a photocatalytic effect. The $\mathrm{SiO}_{2}-\mathrm{TiO}_{2} @$ PDMS hybrid film can be produced on a large scale and the film has high thermal stability at temperatures up to $400{ }^{\circ} \mathrm{C}$. The as-prepared large-scale superhydrophobic cloth was also used as a filter to separate the oil/ water mixture (Fig. 16d). Besides, the "filter cloth" exhibited a considerable separation efficiency with a water contact angle above $150^{\circ}$ and a sliding angle about $8^{\circ}$ after ten times of the separation experiment.

Although the textile products are excellent candidates for preparing absorption materials because of their flexibility, low cost, mechanical stability and high separation efficiency, the application of textile with superhydrophobicity and superoleophilicity for absorbing oils from water is restricted by the inherent poor capacity of fabric. Nowadays, many advanced and feasible technologies to absorb and separate larger amounts of oils or organic pollutants from water have been reported in the literature. Wang et al. ${ }^{134}$ reported a superhydrophobic fabric using a mussel-inspired polydopamine (pDA) and folic acid (FA) to construct a hierarchical structure onto fabric. The strategy did not need any additional nanoparticles and the hierarchical structure was controlled by the concentration of FA or coating time. With the octadecylamine modification, the as-prepared fabric exhibited super-antiwetting properties (Fig. 17a). Taking the energy-saving and high efficiency into consideration, a mini boat formed by fabric was utilized to automatically clean up the oil-spill. $15 \mathrm{~g}$ of crude oil spill was poured onto the water surface and completely absorbed by the boat in ten minutes; meanwhile the crude oil clean-up rate reached as high as $97.1 \%$ (Fig. 17c), showing great potential in environmental remediation for oil spills and organic chemicals. In Liu's work, ${ }^{138}$ a nest-like layered double hydroxide (LDH) microcrystal was in situ grown onto fabric and subsequently modified with low surface energy molecules ensuring the textile with superhydrophobic and superoleophilic properties. Besides the high separation efficiency displayed by the textile membrane, the as-obtained textile was made into a bag filled with a block of sponge. In the absorption experiment, the functional textile acted as a film for the selective absorption of oil from water. The high porosity of sponge acted as a container for storing the penetrated oil. As shown in Fig. 17b(III-VI), when the as-prepared superhydrophobic bag was immersed into the oil/water mixture, the oil was quickly absorbed leaving a clean surface. Importantly, the absorbed oils can be collected by finger squeezing (Fig. $17 \mathrm{~b}$ (VII and VIII)). Similarly, Li et al. ${ }^{145}$ fabricated a continuous and ultrafast absorbing oil bag combining the 2D superhydrophobic and superoleophilic fabric with a large volume capacity of $3 \mathrm{D}$ porous $\mathrm{PU}$ sponge. The superhydrophobic fabric surface was prepared by a sonochemical irradiation method. ${ }^{132}$ The superhydrophobic bag filled with sponge was used for continuous absorption and removal of oil from a series of oil/water mixtures when connected to a vacuum
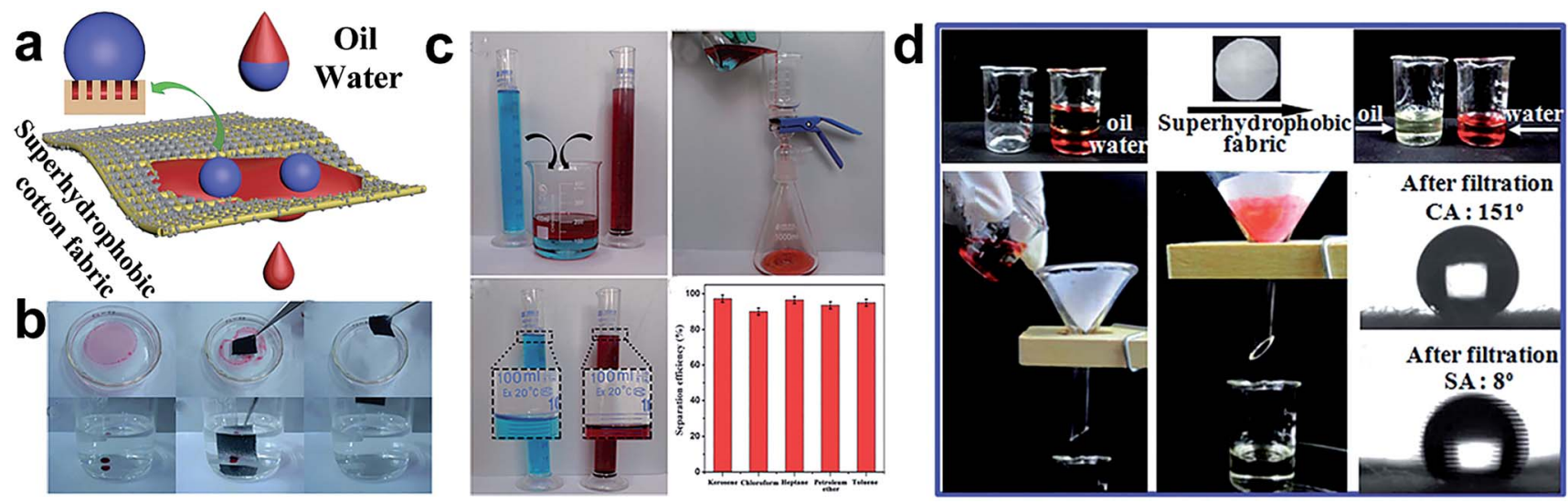

Fig. 16 (a) The scheme illustration of the oil/water separation process. (b) The sequence process of capture of toluene and chloroform from the water surface and underwater by using the as-prepared superhydrophobic and superoleophilic fabric. (c) The experiment of oil/water separation and the separation efficiency of various organic solutions (water dyed with methylene blue and oil dyed with oil red) (reprinted from ref. 132 with permission). (d) The process of oil/water separation by superhydrophobic fabric (the water dyed with alizarine red and the oil used is coal oil) and the images of the water contact angle $(5 \mu \mathrm{L})$ and sliding angle $(10 \mu \mathrm{L})$ after ten separation experiments, respectively (reprinted from ref. 133 with permission). 

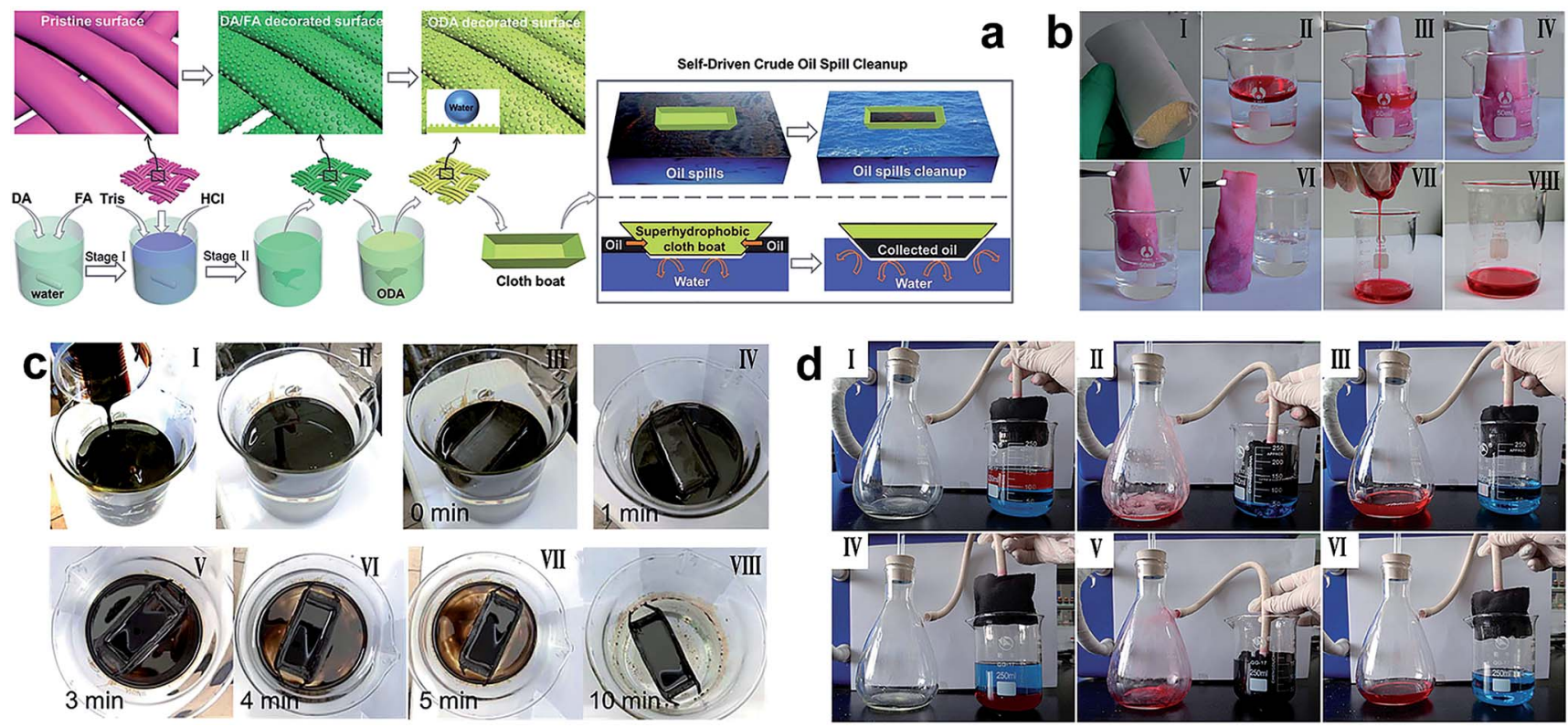

Fig. 17 (a) The schematic illustration of fabricating a microstructure superhydrophobic fabric via a novel mussel-inspired approach and a superhydrophobic cloth boat made for self-driven crude oil spill cleanup. (c) Preparation of oil spill (I and II) and collection process by a superhydrophobic cloth coat (III-VIII) (reprinted from ref. 134 with permission). (b) The sponge wrapped with a superhydrophobic cotton fabric (I) and the resulting superhydrophobic bag absorb and collect organic pollutants (II-VIII) (reprinted from ref. 138 with permission). (d) The continuous absorption and removal oil (dyed with oil red) assisted with a vacuum pump using the superhydrophobic filling bag: absorption and removal of kerosene (I-III) and chloroform (IV-VI) from water (reprinted from ref. 145 with permission).

pump. A separation efficiency higher than $98.2 \%$ and an absorption rate of $3.7 \mathrm{~mL} \mathrm{~cm} \mathrm{~min}^{-1}$ were demonstrated by the 3D PU sponge stuffed into superhydrophobic fabric.

\subsection{Asymmetric/patterning/stimuli-responsive}

An asymmetric surface is an interesting phenomenon in nature, for example, beetle's back and spider silk display two opposite wetting properties, such as superhydrophobic/superhydrophilic, hydrophobic/hydrophilic along different directions. Inspired by this, asymmetric fabric in textile engineering has great potential since one side of the fabric can be made to repel water, oil, and even bacteria and germs, while the other side maintains the intrinsic hydrophilic property. Therefore, the superhydrophobic side provides self-cleaning ability as well as other functional properties and the hydrophilic side keeps the textile moisture absorbing, breathable, comfortable and soft. Besides, the asymmetric or gradient wettability surface allows directional transportation of liquids from the superhydrophobic surface toward the superhydrophilic surface, which has potential in biological, medical, and chemical diagnostic applications. Moreover, the patterned surface with different wettability is also significant to encode chemistry information, to enhance fluid transportation and to enable medical diagnostics.

Liu et al. ${ }^{146}$ reported a single-side superhydrophobic fabric surface by finishing the inherent hydrophilic cotton with fluoropolymer foam. The developed cotton fabric exhibited asymmetric wettability on both sides: one side showing superhydrophobicity and the other maintaining the hydrophilic property. Such asymmetric wettability textile will have great potential in unidirectional liquid transport, microfluidic management, desalination of seawater, and oil/water separation. Zhou et al. ${ }^{147}$ created a superhydrophobic/hydrophilic janus fabric material by a wet-chemical method and successive UV irradiation, demonstrating switchable, spontaneous, directional transport abilities. The coated fabric showed anti-wetting behaviour for both water and oil, and directional transport ability was achieved when the fabric was exposed to UV lamp for a certain duration (Fig. 18a-c). Interestingly, when the fabric exhibited directional transport properties to a liquid solution, it allowed the liquids of lower surface tension to penetrate but refused the penetration of higher surface tension liquids. Moreover, the directional transport ability from one liquid to another can be tuned simply by heating the fabric at higher temperatures and re-irradiating under UV light for an appropriate time. The author also demonstrated that the directional liquid transport ability only relied on liquid surface tension by attaching the liquid droplet ( $45 \mu \mathrm{L})$ on a horizontally laid fabric (Fig. 18d); therefore such membranes are promising for transportation of liquids.

Kwon and co-workers fabricated single-sided superhydrophobic lyocell fabric by oxygen plasma etching for 5 minutes followed by plasma polymerized hexamethyldisiloxane coating for $30 \mathrm{~s}$ (Fig. 18e). ${ }^{148}$ The treated surface of the lyocell transformed into superhydrophobic but the backside showed inherent hydrophilic properties with high moisture absorbing ability (Fig. 18f). Sasaki et al. ${ }^{149}$ adopted one-step spraying a mixture of polymer and $\mathrm{SiO}_{2}$ nanoparticles to fabricate asymmetric wettability cotton fabric. Such a strategy involved 
a

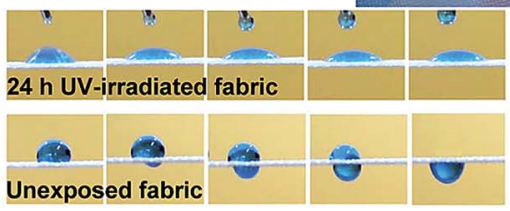

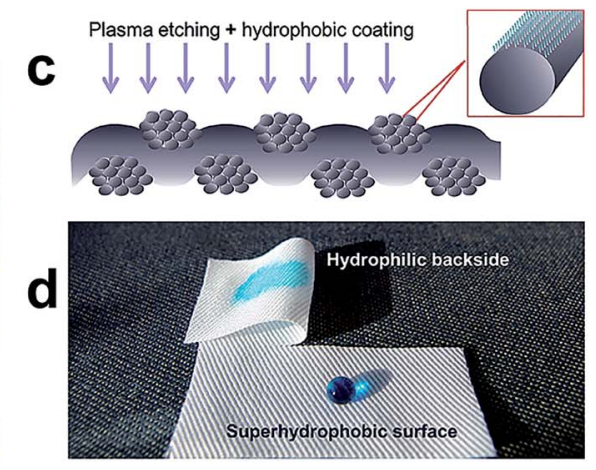

Time
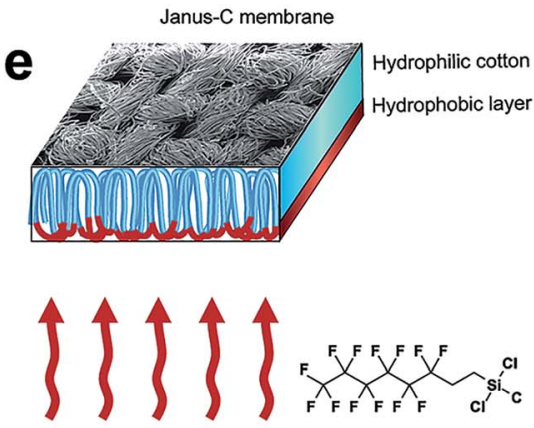

Directional exposure to POTS vapor

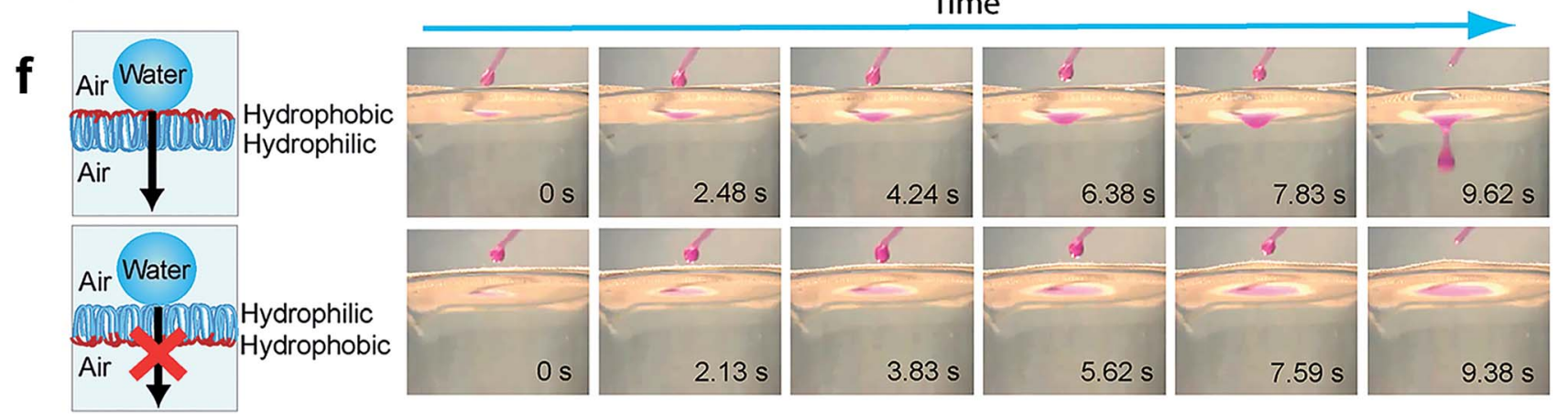

Fig. 18 (a) Photographs of three types of fluid droplets on the resulting janus polyester fabric surface: blue colored water droplet, red colored hexadecane and transparent soybean oil. Scheme of UV irradiation of the asymmetric fabric and water, soybean oil, and hexadecane droplets on the coated fabric surface after exposure for $10 \mathrm{~h}, 14 \mathrm{~h}$, and $24 \mathrm{~h}$. (b) Time sequences of the water penetration process taken from the video on the as-prepared janus fabric surface, top: (UV exposed surface for irradiating $24 \mathrm{~h}$, time interval $0.26 \mathrm{~s}$ ), bottom: (unexposed side, time interval $0.24 \mathrm{~s}$ ). (Reprinted from ref. 147 with permission). (c) Schematic illustration of the plasma-treatment process for preparing a nanostructured fabric surface. (d) Photographic image of the wetting behavior of the water droplet on the asymmetric fabric surface. (Reprinted from ref. 148 with permission). (e) The preparation process of janus fabric when exposed on POTS vapour. (f) Unidirectional droplet penetration illustrated by dropwise addition of the water droplet (dyed with rhodamine 101) on the hydrophobic side (up) and hydrophilic side (bottom) of asymmetric fabric. (Reprinted from ref. 150 with permission).

controlling the asymmetric wettability by altering the distance between the fabric and a sprayer. Besides the asymmetric wettability of the as-designed cotton fabric to water, the asymmetric fabric also showed a similar property to blood. This will be useful for developing multifunctional fabric for medical applications. Generally, the asymmetric surface can be achieved mainly by two routes: (1) to selectively hydrophobize one side of the initially hydrophilic membrane surface and (2) to selectively hydrophilize one side of the inherently hydrophobic surface. These approaches induced different wettability, which benefits directional transportation of liquids. Tian et al. ${ }^{150}$ prepared a macroscopic hydrophilic/hydrophobic janus cotton membrane via a facile vapour diffusion of POTS on one side of cotton fabric (Fig. 16g). The asprepared anisotropic surface chemistry cotton fabric demonstrated directional liquid penetration in an air-water system. As shown in Fig. 16h, a water droplet can penetrate through the janus fabric from the hydrophobic side to the hydrophilic side whereas the water droplet was prevented from going through when placed on the opposite side. This is a clear exhibition of directional droplet gating. They also verified that this directional water penetration across the janus fabric was attributed to its anisotropic critical breakthrough pressure. Such a membrane possessing selective and directional liquid transportation provides a new concept of intelligent materials, which will bring about novel applications in fluid rectifying, microchemical reaction manipulation, biomedicine and smart textile.

The development in multifunctional textiles including liquid/dirt repellent, self-cleaning, UV-shielding, oil/water separation, anti-fouling, photocatalytic, and anti-bacterial/biological has widened their potential application fields. In addition, super-wetting textile materials have other important applications in micro-pattern regulation, stimuli-reflection, gas detection, etc. Wang et al. ${ }^{151}$ fabricated a hydrophilically patterned superhydrophobic cotton fabric via in situ synthesis of a block copolymer and subsequently photolyzing the cotton swatch to anchor a poly[2-(cinnamoyloxy)ethyl acrylate] (PCEA) block crosslink in the exposed area. After extracting the cotton swatch with $\mathrm{CH}_{2} \mathrm{Cl}_{2}$, a hydrophilic pattern assembled in the masked area, and the exposed area was superhydrophobic (Fig. 19a-d). In addition, the photographic patterns were printed on cotton and polyester/cotton surface using diluted ink and PEG-containing ink. Experiments indicated that an increase in viscosity of ink could eliminate the bulging effect caused by the good wetting ability of cotton (Fig. 19e and f). Thus, such hydrophilically patterned superhydrophobic cotton fabric can be used as stamps for ink or dye printing. A bioinspired, stimuli- 
response, multifunctional superhydrophobic surface was developed by self-assembly of a graphene monolayer and $\mathrm{TiO}_{2}$ nanofilm. The micro/nanostructure provided by a graphene monolayer and $\mathrm{TiO}_{2}$ nanofilm on fabric (GT-fabric) was shown to have enhanced surface roughness and superhydrophobicity. ${ }^{152}$ Some smart surface characters, such as tunable adhesion, wettability and directional water transportation, can be obtained upon UV irradiating the upper $\mathrm{TiO}_{2}$ layer. The GT-fabric can be used as a novel droplet sensor when loaded with different initiators for accurate sensing of certain gases. For example, the droplet array was loaded with different types of initiators, such as $\mathrm{Cu}^{2+}$ (Fig. 19g and h), congo red (Fig. 19i and j), and $\mathrm{Pd}^{2+}$ (Fig. 19k and l) for sensing $\mathrm{NH}_{3}, \mathrm{HCl}$, and ethylene diamine. The water droplet was stable on the GT-fabric surface even at a tilt angle of $90^{\circ}$ and $180^{\circ}$ (Fig. 19n-p) because of the high contact angle and high adhesion, exhibiting a typical "rose petal" effect. Such smart superhydrophobic GT-fabric can also achieve droplet manipulations, including storage, transfer and mixing (Fig. 19q and r). On the other hand, carbon nanotubes can be easily removed by a water droplet as a result of robust superhydrophobic on the GTfabric surface (Fig. 19t-w).

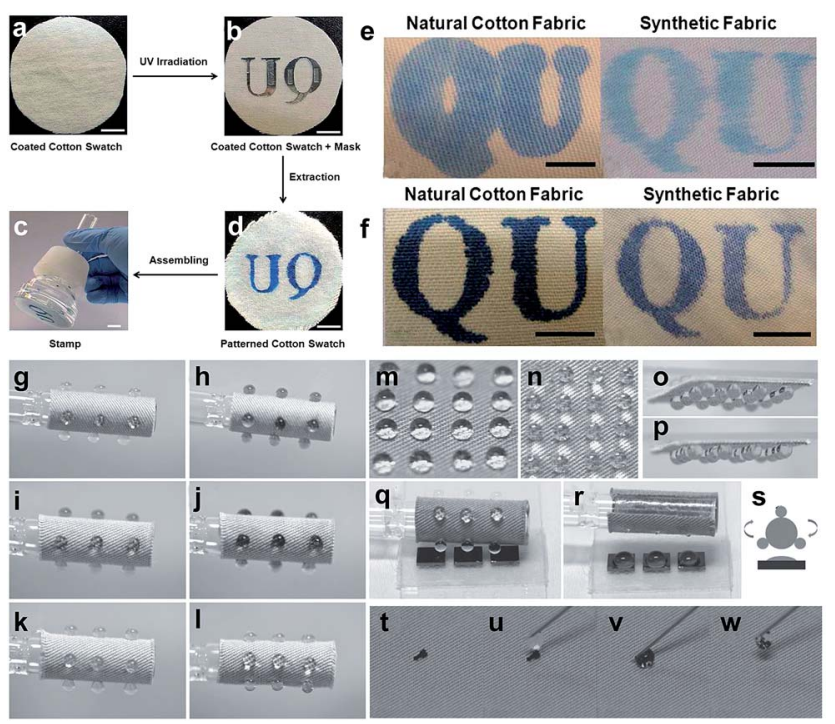

Fig. 19 The preparation process of the cotton-based stamp. (a) Immersing the cotton stamp into a polymer solution and taking it out from the solution and annealing the cotton yielded a polymer coated cotton surface. (b) The coated cotton stamp was covered with an aluminium mask and irradiated. (c) A hydrophilic patterned cotton swatch prepared after extracting with $\mathrm{CH}_{2} \mathrm{Cl}_{2}$. (d) The hydrophilic patterned cotton fabric attached to a funnel to make a stamp for inkprinting. The patterns of "QU" are printed by using diluted ink (e) and PEO-containing ink (f) onto cotton and polyester/cotton (65\%/35\%) blending fabric. (Reprinted from ref. 138 with permission). The droplet sensors based on the flexible GT surface with various initiators: ( $g$ and $\mathrm{h}$ : $\mathrm{Cu}^{2+} ; \mathrm{i}$ and $\mathrm{j}$ : congo red; $\mathrm{k}$ and $\mathrm{l}: \mathrm{Pd}^{2+}$ ) for different stimuli ( $\mathrm{g}$ and $\mathrm{h}$ : $\mathrm{NH}_{3} ; \mathrm{i}$ and $\mathrm{j}$ : $\mathrm{HCl} ; \mathrm{k}$ and $\mathrm{l}$ : ethylene diamine). Water droplets arranged with a $4 \times 4$ array were stored on the GT fabric surface $(\mathrm{m})$ which remain stable when tilting the sample to $90^{\circ}(\mathrm{n})$ and $180^{\circ}(\mathrm{o}, \mathrm{p})$. (q-s) the transfer process of the water droplet from GT fabric to a silicon wafer. $(t-w)$ the self-cleaning property of GT fabric: carbon nanotube powder was removed from the fabric surface using a water droplet. (Reprinted from ref. 152 with permission).

\subsection{Durability/self-healing/UV-shielding/flame-retardant}

Superhydrophobic fabrics are flexible, air-breathing, and even superamphiphobic, making them potentially useful as selfcleaning cloths. However, the applications of such fabrics are restricted by their poor stability and durability since once being mechanically and/or chemically stressed, they will lose their superhydrophobic property. ${ }^{153,154}$ From a practical point of view, it is indispensable to develop robust superhydrophobic surfaces with good laundering and mechanical abrasion properties to cater for the increasing consumers' needs and market demands. There are generally two routes to construct robust and durable superhydrophobic fabric surfaces. One is to create roughness and establish the chemical bond between the coating and fabric; ${ }^{155-162}$ The other is to introduce the bioinspired self-healing function into the coated fabric..$^{10,83,112,113,163-169}$ Many research studies have been reported to fabricate a robust superhydrophobic fabric surface with durability and stability against laundering, mechanical abrasion, and chemical attack. For example, Deng et al. ${ }^{155}$ reported a radiation-induced graft polymerization method to obtain an extremely stable and superhydrophobic cotton fabric surface, exhibiting excellent chemically stability over the entire range $\mathrm{pH}$ (0-14) and laundering durability for 250 commercial washing cycles. The fluorinated fabric still maintained superhydrophobicity after 50 accelerated laundering cycles (Fig. 20a and $b$ ), which indicates that the binding between the cotton fibre and fluorinated polymer chain was strong enough to withstand the shear force of water and stainless steel balls. Abbas et al. $^{\mathbf{1 5 6}}$ used ethylenediaminetetraacetic acid (EDTA) to alternate the silane coupling agent as a crosslinking in the presence of silica nanoparticles (SNPs) and hexadecyltrimethoxysilane (HDTMS) to fabricate improved laundering durability superhydrophobic fabric. In addition, in Das's work, ${ }^{161}$ a extremely durable superhydrophobic coating was fabricated by a sol-gel method and the fabric surface was firmly attached to the fluorinated coating through covalent bonding by a simple immersion technology. The coated fabric retained its superhydrophobicity with a high water contact angle of $161^{\circ}$ and a low contact angle hysteresis of $4^{\circ}$ after several cycles of the sand paper abrasion test, demonstrating that the covalent bonding between the coating and cotton fibre has been established (Fig. 20c).

Xue et al. $^{\mathbf{1 6 2}}$ reported a washable and wear-resistant superhydrophobic textile by chemically etching the microscale fibre with an alkali solution, followed by coating with polydimethylsiloxane (PDMS) (Fig. 20d). The obtained fabric showed self-cleaning properties and also robust to alkali/acid etching, UV-irradiation, and long-time laundering. Furthermore, the PDMS-E-PET fabric still remained superhydrophobic on the broken area after abrasion of 3000 cycles (Fig. 20e). Importantly, colourful images can be imparted on the superhydrophobic surface by using conventional dyeing or thermal transfer printing without affecting the antiwettability (Fig. 20f). Wu et al. ${ }^{163}$ developed a colourful self-healing and durable antibacterial superhydrophobic surface via a solution dipping method using sequential deposition of poly(ethylenimine) 

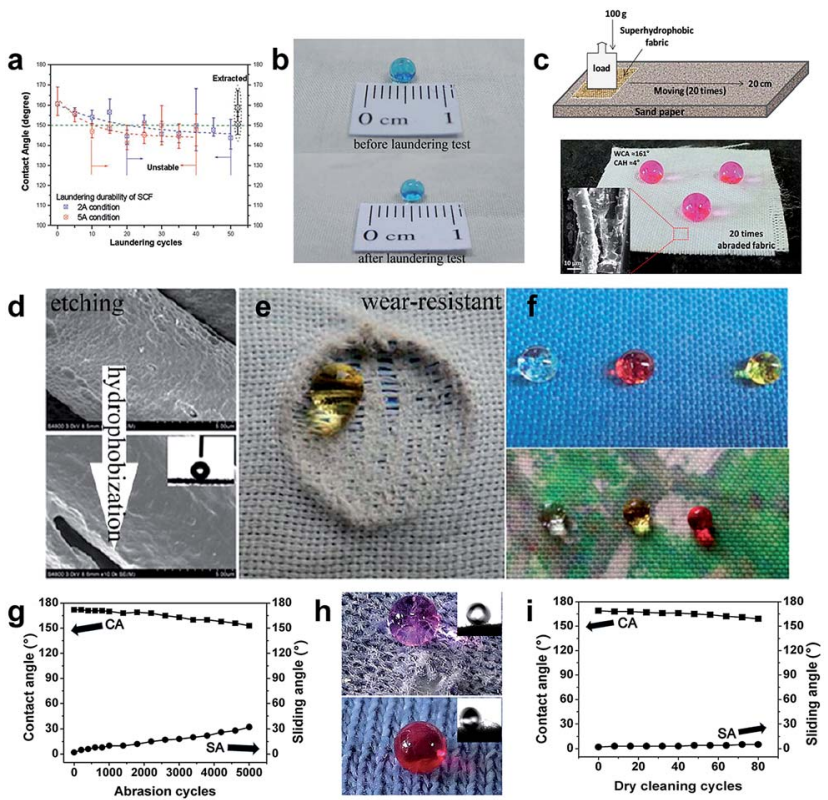

Fig. 20 (a) The relationship between the water contact angle and laundering cycles for the superhydrophobic cotton fabric with a DG of $27.3 \%$ under $2 \mathrm{~A}$ and $5 \mathrm{~A}$ conditions according to the AATCC 61-2006 standard method. (b) The images of the water droplet before and after the laundering test. (Reprinted from ref. 155 with permission). (c) The schematic illustration of the abrasion test of coated fabric on sand paper and the photograph of the dyed water droplet on the coated fabric for 20 times abrasion; the inset is the surface morphology of the abraded coating surface (20 times). (Reprinted from ref. 161 with permission). (d) The surface structures on PET fabric after etching and hydrophobized with PDMS. (e) A water droplet on a broken area of PDMS-E-PET after abrasion for 3000 cycles. (f) Water droplets were stable on the coloured and printed fabric surface, indicating that conventional dyeing or thermal printing has no influence on superhydrophobicity. (Reprinted from ref. 162 with permission). (g) The changes of the contact angle and sliding angle of F-POSS/AgNPs/PEIcoated cotton fabric undergoing 5000 abrasion cycles. (h) Dyed water droplet located on the F-POSS/AgNPs/PEI-coated cotton fabric surface for 5000 cycles of abrasion and healing (upper) and 80 cycles of dry cleaning and healing (bottom); the insets are the images of the sliding angle, respectively. (i) The changes of the contact angle and sliding angle of F-POSS/AgNPs/PEI-coated cotton fabric within 80 cycles of dry cleaning. (Reprinted from ref. 163 with permission).

(PEI), silver nanoparticles (AgNPs), and fluorinated decyl polyhedral oligomeric silsesquioxane (F-POSS). The F-POSS/AgNPs/ PEI coated fabric showed remarkable resistance to abrasion and dry-cleaning, and the water contact angle and sliding angle changed to about $152^{\circ}$ and $32^{\circ}$ after being subjected to 5000 cycles of abrasion. The as-prepared fabric was still superhydrophobic after 80 cycles of the dry-cleaning process with a high water contact angle of around $159^{\circ}$ and a low sliding angle of $5^{\circ}$ (Fig. $20 \mathrm{~g}-\mathrm{i}$ ).

The self-healing superhydrophobic surface can restore its water repellent property after being destroyed by acid, base, chemical reagents, and mechanical and laundering abrasion. Generally, the destroyed surface is repaired to original superhydrophobic by heating treatment, humid environmental conditioning, and ironing treatment and so on. Zhou et al. ${ }^{113}$ reported a two-step wet-chemistry coating method for durable self-healing superamphiphobic surfaces. The coated fabric exhibited excellent durability to acid, UV light, mechanical and washing abrasion. After being damaged, the fabric can restore its super liquid-repellent performance by a short-time heating or room temperature ageing. Xi et al. ${ }^{164}$ described a simple onepot mist copolymerization technology to construct healable superhydrophobic fabric. Moreover, the modified cotton fabric surface can recover its superhydrophobicity by ironing treatment after 60 cycles of laundering or 2000 times of Martindale abrasion. A flame-retardant and self-healing superhydrophobic coating was successfully obtained onto the cotton fabric surface via the solution-dipping method. ${ }^{165}$ After etching with $\mathrm{O}_{2}$ plasma, the resulting cotton surface was superhydrophilic (Fig. 21a). The coating can repetitively and spontaneously restore the superhydrophobicity by placing the damaged cotton fabric in a humid environment with a relative humidity of $35 \%$ for about one hour (Fig. 21b). Similarly, the F-POSS/AgNPs/PEIcoated cotton fabric surface was fully superhydrophilic when exposed to $\mathrm{O}_{2}$ plasma; however, it can recover its original superhydrophobic state under an ambient environment at $25^{\circ} \mathrm{C}$ and a relative humidity ( $\mathrm{RH}$ ) of $55 \%$. The etching/healing process can be repeated for at least 16 cycles without apparent changes, showing a strong healing ability of damaged superhydrophobic fabric. ${ }^{163}$ Most reported self-healing surfaces are achieved by migrating low surface energy molecules into the damaged surface to recover the special wettability.

Though much literature reported on self-healing coating with extreme wettability, the usage of fluorine-containing agents is harmful for human body and environment. From this perspective, it is essential to create liquid repellent coating with long-term durability, self-healing and non-toxicity, which is believed to be an efficient way to overcome the poor durability caused by physical and chemical damages. Zhou et al. ${ }^{166}$ reported a new approach to construct self-healing super-wettability without any fluorine-containing agents. In this work, polydopamine@octadecylamine (PDA@OCA) nanocapsules were used as coating materials and added onto fabric surface by using an in-situ polymerization method. When the coated fabric was destroyed and lost its liquid repellency, the OCA molecules could migrate to fabric surface and restore its wettability only on heating treatment. For illustration, the coated fabric was treated with $\mathrm{O}_{2}$ plasma and the surface was superhydrophilic with contact angles of almost $0^{\circ}$ for any liquids (Fig. 21d). Interestingly, when the fabric treated by plasma was heated at $80{ }^{\circ} \mathrm{C}$, its liquid repellency recovered (Fig. 21f). Moreover, the self-healing can be repeated more than 10 times. The contact angles for various liquids (water, juice, coffee and milk) on coated fabric surface after 10 cycles of plasma and heat curing indicated that its self-healing property can be easily obtained by simple heating (Fig. 21h).

\subsection{Other potential applications}

Through the past research, human beings have learnt insightful knowledge from nature and managed to fabricate multifunctional textile materials mimicking the lotus leaf and others. The multifunctional superhydrophobic surfaces possess many 

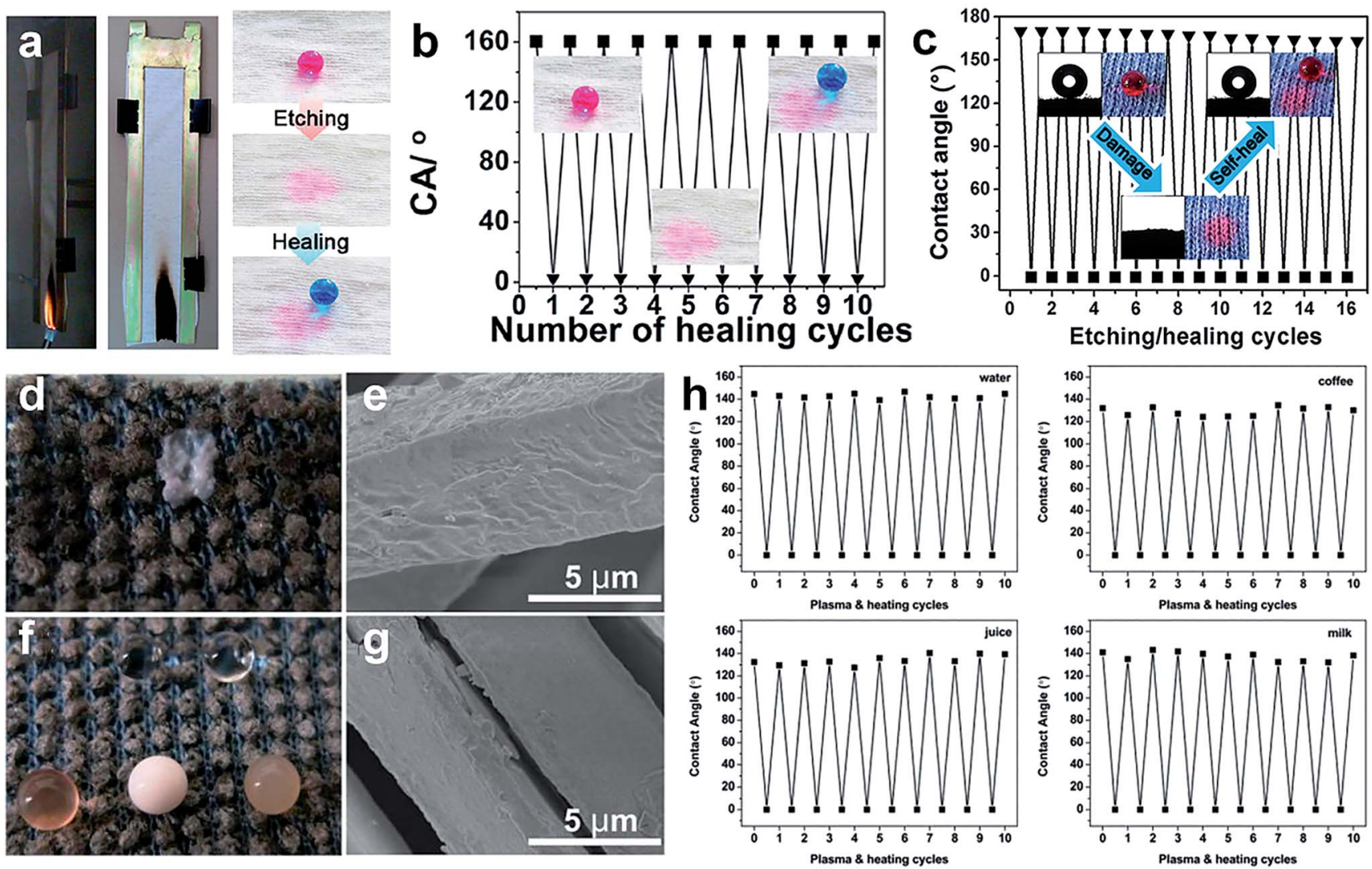

Fig. 21 (a) The flame-retardant and self-healing superhydrophobic fabric coated by a tri-layer of branched poly(ethylenimine) (bPEI), ammonium polyphosphate (APP), and fluorinated-decyl polyhedral oligomeric silsesquioxane (F-POSS). (b) The water contact angle on F-POSS/APP/bPEI coated fabric after $\mathrm{O}_{2}$ plasma etching and healing for 10 cycles. (Reprinted from ref. 165 with permission). (c) The water contact angle changes on F-POSS/AgNPs/bPEI coated fabric after $\mathrm{O}_{2}$ plasma etching and healing for 10 cycles. (Reprinted from ref. 163 with permission). Photographs of liquid droplets on fabric after plasma treatment (d) and plasma and heat curing (f), and the SEM images of the fabric surface after plasma treatment (e) and plasma and heat curing (g). (h) The contact angles of water, juice, coffee, and milk on the coated fabric surface for 10 cycles of plasma and heat treatment. (Reprinted from ref. 166 with permission).

significant properties including anti-wetting (liquids, dirt, and emulsions), self-cleaning, air-transport, UV-shielding, corrosion-resistance, antibacteria, flame-retardance and so on. Other important applications include oil/water separation, photocatalysis, self-healing, and microfluidic management. In addition, superhydrophobic fabrics have good potential in water/fog collection, anti-icing and electromagnetic interference shielding. Wang et al. ${ }^{170}$ developed a superhydrophobic surface with a light-induced superhydrophilic area inspired by 1D spider silk and 2D beetle back with efficient water-collecting properties. The fabrication process involved construction of a superhydrophobic fabric surface via incorporation of surface roughness and low surface energy. Subsequently, the superhydrophobic fabric was sprayed with $\mathrm{TiO}_{2}$ nanosol to induce superhydrophilic bumps under light irradiation (Fig. 22a). The $\mathrm{TiO}_{2}$ domains could be observed on the superhydrophobic substrate (Fig. 22b). Their presence has created both a wettability gradient and shape gradient, which is very significant to accelerate water coalescence and water collection. During the fog condensation process, there is no spherical water droplet observed on fabric sprayed with $\mathrm{TiO}_{2}$ nanosol (the cotton fabric treated with only $\mathrm{TiO}_{2}$ was defined as TC fabric). However, visible spherical water droplets were formed on SSC (the cotton fabric treated with $\mathrm{SiO}_{2}$ NPs and $n$-octadecyltriethoxysilane) and SSTC-11 (the fabric treated with $\mathrm{SiO}_{2} \mathrm{NPs}, n$-octadecyltriethoxysilane and $\mathrm{TiO}_{2}$ nanosol with a spraying distance of 11 $\mathrm{cm})$ fabric surface, more and larger coalesced water droplets were collected on the SSTC-11 fabric surface due to the presence of superhydrophilic sites compared with the SSC superhydrophobic surface (Fig. 22e). The above results indicated that the introduction of superhydrophilic $\mathrm{TiO}_{2}$ nanosol onto the originally superhydrophobic fabric surface was beneficial to enhance the efficiency of water-collection.

Zou et al. ${ }^{171}$ reported a superhydrophobic cotton surface with durable electromagnetic interference shielding. The specific process is shown in Fig. 22c, and cotton fabric was deposited on a coating solution containing of Nafion and multiwalled carbon nanotubes (MWCNTs), in which Nafion was used as a linker between the pristine cotton fibre and hydrophobic MWCNTs, meanwhile providing a homogenous dispersion. After 6 cycles of the deposition process, the cotton fabric coated with NafionMWCNTs exhibited a water contact angle of $154.6^{\circ}$ and a favourable electromagnetic interference shielding effectiveness of $0.9 \mathrm{~dB}$ (Fig. 22f). Moreover, the resultant fabric also 
a
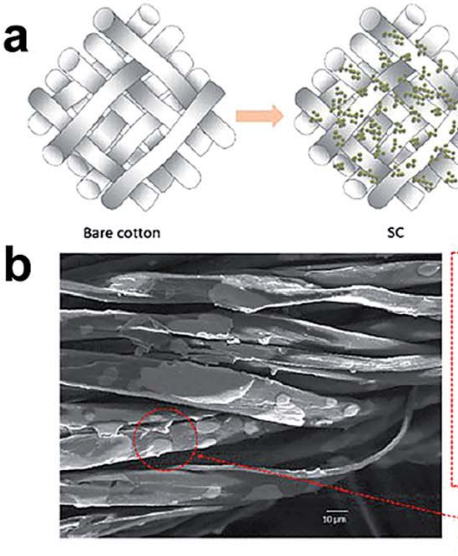

sc
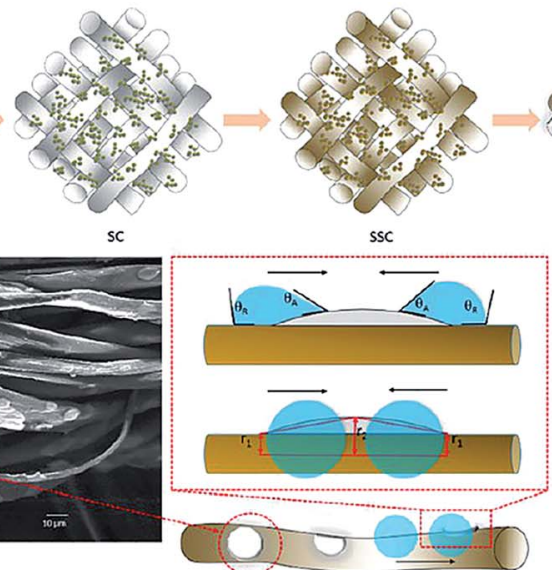

e

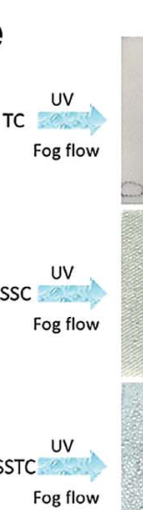

$1 \mathrm{~min}$

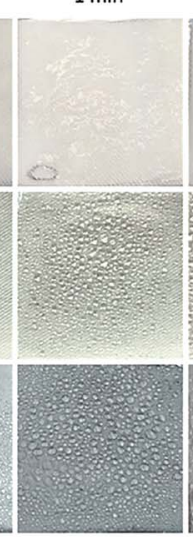

$2 \min$

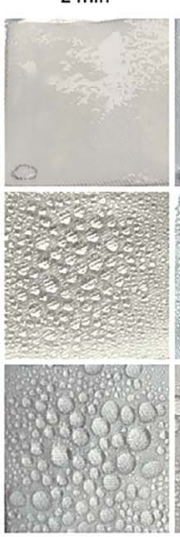

$3 \mathrm{~min}$

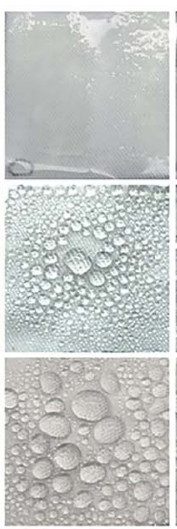

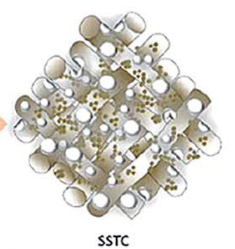

SSTC

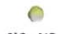

$\mathrm{SiO}_{2} \mathrm{NPS}$

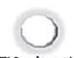

$\mathrm{TiO}_{2}$ domains

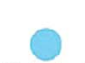

Water droplet

$4 \mathrm{~min}$

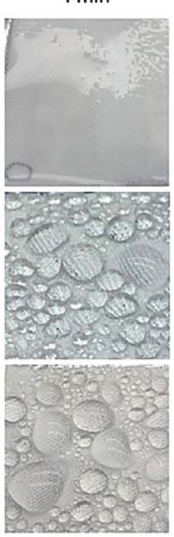

C

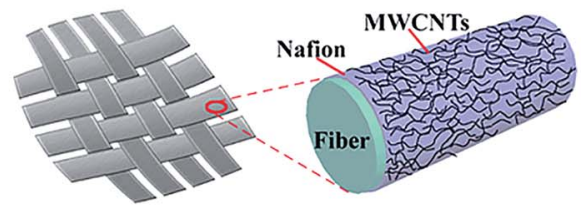

Nafion:
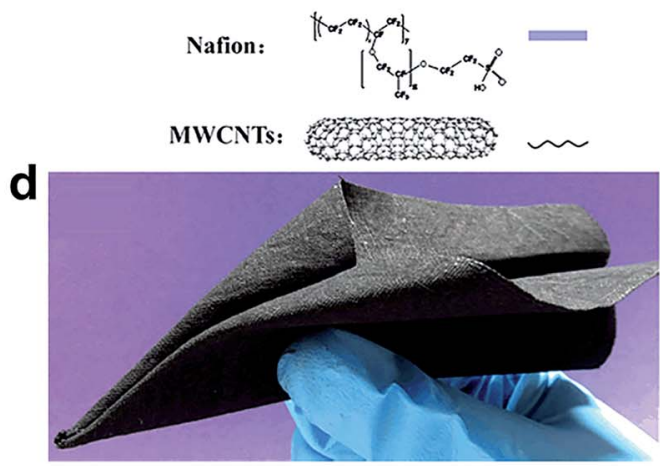

f

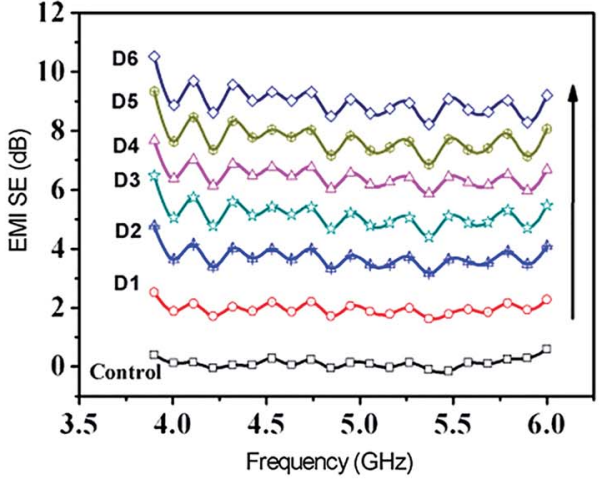

Fig. 22 (a) Illustration of the fabrication process for SC, SSC, and SSTC fabrics. (b) The SEM image of SSTC-11 fabric in which the red square represents the raised $\mathrm{TiO}_{2}$ domains (left) and the schematic illustration of the driving force as well as Laplace pressure between the superhydrophobic fibre and hydrophilic $\mathrm{TiO}_{2}$ area (right). (e) Water condensation and coalescence process on TC, SSC, SSTC fabric surface with time going on. (Reprinted from ref. 170 with permission). (c) Schematic of the preparation of superhydrophobic, durable electromagnetic interference shielding cotton fabrics and (d) photograph of the as-prepared cotton fabric coated with Nafion and Multiwalled Carbon Nanotubes (MWCNTs). (f) Electromagnetic interference shielding (EMI SE) of fabrics with different layer deposition. (Reprinted from ref. 171 with permission).

possessed good durability in electromagnetic interference shielding after soaking in water for 4 days or washing with the American Association of Textile Chemists and Colourists (AATCC) standard (61-2013 test no. 2A) due to its excellent superhydrophobicity and chemical stability.

Another attractive application of superhydrophobic surfaces, in addition to the extraordinary water-repellence, is their excellent capability to reduce accumulation of snow and ice and to even completely prevent ice formation in a low humidity environment. Recently, numerous studies of superhydrophobic coating on rigid metal substrates demonstrated lower adhesions to both liquid water droplets and ice than bare metals or metals covered with hydrophobic coating. ${ }^{172-187}$ The anti-icing properties of superhydrophobic surfaces have great potential applications in aircrafts, optical lenses, energy transmission system, power lines, wind turbines, and highways as well as building constructions. ${ }^{188-196}$ However, reports on potential anti-icing application by flexible textile surfaces are very rare. Lee et $a{ }^{197}$ reported the anti-icing property of industrial nonwoven geo-textiles, which exhibited de-icing and anti-icing properties compared to the controls. They also found that surface morphologies as well as surface tension of the substrate have a great effect on anti-icing properties.

\section{Conclusions and future prospects}

In this review, we summarize characterization methods, fabrication technologies, and multifunctional applications of special wettability textile surfaces. These super-wettability flexible textile surfaces possess significant value to both fundamental research and practical applications. Inspired by the nature, great achievements have been made in this field. Various superantiwetting surfaces have been fabricated by combining the design of surface roughness and surface chemistry. ${ }^{198-207}$ The fabrication strategies reported in the literature can be mainly divided into two categories: pre-roughening and post-fluorinating, and roughening and fluorinating at the same time. From this point of consideration, the fabrication technologies are described briefly in this review. The likely and potential applications are also reviewed including superamphiphobicity, self-cleaning, oil/water separation, UV-shielding, anti-bacterial/ 
biological implant, sensor, asymmetric/janus fabric, patterning, and self-healing surface. ${ }^{208-220}$

Although great achievements have been made, there are still difficulties and challenges that need to be overcome. From the fundamental research point of view, there is still a lack of the basic mechanism on the interactions between textile and chemical reagents during the fabrication process. Besides, the majority of fabrication methods are limited to laboratory and not suitable for industrial production on a large scale. And although many highlighted super-antiwettability textiles have been obtained, they may suffer from poor mechanical stability and durability during laundering and so on. The mechanical durability of water-repellence fabric is very crucial to practical products. Some developed durable mechanical superhydrophobic fabrics have been reported in the literature; however, the relationship between the durability and surface morphology as well as surface chemistry remains unclear and should be researched in-depth in the future. Moreover, it is necessary to conduct and develop a series of evaluation methods for superhydrophobic textile materials, considering these textured structures are different from conventional solid materials. For example, the laundering test may have to take into consideration the resulting fabric size, weight per square meter, and material species. The evaluation of the self-cleaning effect is another area to be standardized. So far in the literature, the test methods and the type of pollutant used vary a lot, making it difficult to compare among different reported results.

In most reports, fluoro-containing compounds are introduced into the surfaces due to their low surface energy. It should be noted that most of the fluoro-containing compounds are toxic and harmful to human health. Thus the use of fluorocontaining chemical reagents should be eliminated or at least minimized. The safety verification of the used nano-particles and the low surface energy compounds should also be carried out before scalable production can be made.

Overall, many interesting, remarkable, and meaningful studies have been revealed for multifunctional super-antiwettability surfaces based on flexible and inexpensive textile substrates. Among these functional properties, durability against physical and chemistry damages is an important consideration for the sustainable development and application, and more efforts are clearly needed. The future trend is to develop durable self-healing superhydrophobic surfaces that can automatically restore original characteristics after having been damaged. Controllable superhydrophobic surfaces with various surface energies may be another research hotspot, which have great potential in the biology-related field. There is undoubtedly a bright future for the development of superwettability textiles and we wish this review could provide some thoughts for potential strategies that will benefit researchers and engineers in this field.

\section{Acknowledgements}

The authors acknowledge the National Natural Science Foundation of China (21501127; 51502185), Natural Science Foundation of Jiangsu Province of China (BK20140400), Natural
Science Foundation of the Jiangsu Higher Education Institutions of People's Republic of China (15KJB430025), Priority Academic Program Development of Jiangsu Higher Education Institutions (PAPD), and Project for Jiangsu Scientific and Technological Innovation Team (2013) for financial support of this work.

\section{Notes and references}

1 H. Ollivier, Ann. Chim. Phys., 1907, 10, 229.

2 W. Barthlott and C. Neinhuis, Planta, 1997, 202, 1.

3 X. Zhang, F. Shi, J. Niu, Y. Jiang and Z. Wang, J. Mater. Chem., 2008, 18, 621.

4 K. Koch, B. Bhushan and W. Barthlott, Prog. Mater. Sci., 2009, 54, 137.

5 H. J. Wang, Z. Yang, J. Yu, Y. Z. Wu, W. J. Shao, T. T. Jiang and X. L. Xu, RSC Adv., 2014, 4, 33730.

6 A. Milionis, D. Fragouli, L. Martiradonna, G. C. Anyfantis, P. D. Cozzoli, I. S. Bayer and A. Athanassiou, ACS Appl. Mater. Interfaces, 2014, 6, 1036.

7 D. S. Facio and M. J. Mosquera, ACS Appl. Mater. Interfaces, 2013, 5, 7517.

8 T. Verho, C. Bower, P. Andrew, S. Franssila and O. Ikkala, Adv. Mater., 2011, 23, 673.

9 N. L. Tarwal, V. M. Khot, N. S. Harale, S. A. Pawar, S. B. Pawar, V. B. Patil and P. S. Patil, Surf. Coat. Technol., 2011, 206, 1336.

10 J. X. Wu, J. Y. Li, B. Deng, H. Q. Jiang, Z. Q. Wang, M. Yu, L. F. Li, C. Y. Xing and Y. J. Li, Sci. Rep., 2013, 3, 2951.

11 B. Cortese, D. Caschera, F. Federici, G. M. Ingo and G. Gigli, J. Mater. Chem. A, 2014, 2, 6781.

12 J. Y. Huang, S. H. Li, M. Z. Ge, L. N. Wang, T. L. Xing, G. Q. Chen, X. F. Liu, S. S. Al-Deyab, K. Q. Zhang, T. Chen and Y. K. Lai, J. Mater. Chem. A, 2015, 3, 2825.

13 J. Li, L. Shi, Y. Chen, Y. B. Zhang, Z. G. Guo, B. L. Su and W. M. Liu, J. Mater. Chem., 2012, 22, 9774.

14 X. Liu, L. Ge, W. Li, X. Wang and F. Li, ACS Appl. Mater. Interfaces, 2015, 7, 791.

15 E. A. Vogler, Adv. Colloid Interface Sci., 1998, 74, 69.

16 C. W. Guo, S. T. Wang, H. Liu, L. Feng, Y. L. Song and L. J. Jiang, J. Adhes. Sci. Technol., 2008, 22, 395.

17 A. Lafuma and D. Quere, Nat. Mater., 2003, 2, 457.

18 N. A. Patankar, Langmuir, 2004, 20, 7097.

19 S. Wang and L. Jiang, Adv. Mater., 2007, 19, 3423.

20 L. Feng, S. H. Li, Y. S. Li, H. J. Li, L. J. Zhang, J. Zhai, Y. L. Song, B. Q. Liu, L. Jiang and D. B. Zhu, Adv. Mater., 2002, 14, 1857.

21 Y. M. Zheng, X. F. Gao and L. Jiang, Soft Matter, 2007, 3, 178.

22 K. Autumn, M. Sitti, Y. C. A. Liang, A. M. Peattie, W. R. Hansen, S. Sponberg, T. W. Kenny, R. Fearing, J. N. Israelachvili and R. J. Full, Proc. Natl. Acad. Sci. U. S. A., 2002, 99, 12252.

23 K. Autumn, Y. A. Liang, S. T. Hsieh, W. Zesch, W. P. Chan, T. W. Kenny, R. Fearing and R. J. Full, Nature, 2000, 405, 681.

24 H. Lee, B. P. Lee and P. B. Messersmith, Nature, 2007, 448, 338. 
25 G. McHale, N. Shirtcliffe and M. Newton, Langmuir, 2004, 20, 10146.

26 N. A. Patankar, Langmuir, 2003, 19, 1249.

27 A. Marmur, Langmuir, 2003, 19, 8343.

28 W. A. Zisman, Ind. Eng. Chem., 1963, 55, 18.

29 A. Sengupta, S. N. Malik and D. Bahadur, Appl. Surf. Sci., 2016, 363, 346.

30 P. Aminayi and N. Abidi, Surf. Coat. Technol., 2015, 276, 636.

31 Q. Zhao, L. Y. L. Wu, H. Huang and Y. C. Liu, Mater. Des., 2016, 92, 541.

32 H. Teisala, M. Tuominen and J. Kuusipalo, Adv. Mater. Interfaces, 2014, 1, 1300026.

33 X. Zhang, F. Shi, J. Niu, Y. Jiang and Z. Wang, J. Mater. Chem., 2008, 18, 621.

34 Z. L. Chu and S. Seeger, Chem. Soc. Rev., 2014, 43, 2784.

35 J. Zimmermann, M. Rabe, G. R. J. Artus and S. Seeger, Soft Matter, 2008, 4, 450.

36 G. R. J. Artus, S. Jung, J. Zimmermann, H. P. Gautschi, K. Marquardt and S. Seeger, Adv. Mater., 2006, 18, 2758.

37 H. X. Wang, H. Zhou, G. Adrian, J. Fang and T. Lin, ACS Appl. Mater. Interfaces, 2013, 5, 10221.

38 B. Xu, Y. Y. Ding, S. B. Qu and Z. S. Cai, Appl. Surf. Sci., 2015, 356, 951.

39 H. B. Hu, L. Gao, C. L. Chen and Q. W. Chen, Environ. Sci. Technol., 2014, 48, 2928.

40 T. Makowski, D. Kowalczyk, W. Fortuniak, D. Jeziorska, S. Brzezinski and A. Tracz, Cellulose, 2014, 21, 4659.

41 I. S. Bayer, D. Fragoul, A. Attanasio, B. Sorce, G. Bertoni, R. Brescia, R. di Corato, T. Pellegrino, M. Kalyva and S. Sabella, ACS Appl. Mater. Interfaces, 2011, 3, 4024.

42 D. Xiong and G. J. Liu, Langmuir, 2012, 28, 6911.

$43 \mathrm{H}$. Hu, G. J. Liu and J. Wang, Adv. Mater. Interfaces, 2016, 3, 1600001.

44 D. Xiong, G. J. Liu and L. Z. Hong, Chem. Mater., 2011, 23, 4357.

45 X. Wu, I. Wyman, G. W. Zhang, J. Lin, Z. Q. Liu, Y. Wang and $\mathrm{H}$. Hu, Prog. Org. Coat., 2016, 90, 463.

46 H. X. Wang, H. Zhou, W. D. Yang, Y. Zhao, J. Fang and T. Lin, ACS Appl. Mater. Interfaces, 2015, 7, 22874.

47 S. H. Li, J. Y. Huang, M. Z. Ge, C. Y. Cao, S. Deng, G. Q. Chen, K. Q. Zhang, S. S. Al-Deyab and Y. K. Lai, Adv. Mater. Interfaces, 2015, 2, 1500220.

48 J. W. Zeng, B. Wang, Y. B. Zhang, H. Zhu and Z. G. Guo, Chem. Lett., 2014, 43, 1566.

49 M. Zhang, D. L. Zang, J. Y. Shi, Z. X. Gao, C. Y. Wang and J. Li, $R S C A d v ., 2015$, 5, 67780.

50 Y. S. Joung and C. R. Buie, ACS Appl. Mater. Interfaces, 2015, 7, 20100.

51 M. Cakir, I. Kartal and Z. Yildiz, Text. Res. J., 2014, 84, 1528.

52 Y. K. Lai, Y. X. Tang, J. J. Gong, D. G. Gong, L. F. Chi, C. J. Lin and Z. Chen, J. Mater. Chem., 2012, 22, 7420.

53 N. Nuraje, W. S. Khan, Y. Lei, M. Ceylan and R. Asmatulu, J. Mater. Chem. A, 2013, 1, 1929.

54 Y. T. Luo, J. Li, J. Zhu, Y. Zhao and X. F. Gao, Angew. Chem., Int. Ed., 2015, 54, 4876.

55 Z. L. Chu and S. Seeger, RSC Adv., 2015, 5, 21999.
56 B. C. Li, J. P. Zhang, L. Wu and A. Q. Wang, ChemPlusChem, 2013, 78, 1503.

57 B. J. Spark, E. F. T. Hoff, L. Xiong, J. T. Goetz and D. L. Patton, ACS Appl. Mater. Interfaces, 2013, 5, 1811.

58 W. Duan, A. J. Xie, Y. H. Shen, Y. F. Wang, F. Wang, Y. Zhang and J. L. Li, Ind. Eng. Chem. Res., 2011, 50, 4441.

59 H. X. Wang, J. Ding, Y. H. Xue, X. G. Wang and T. Lin, J. Mater. Res., 2010, 25, 1336.

60 Q. W. Gao, Q. Zhu, Y. L. Guo and C. Q. Yang, Ind. Eng. Chem. Res., 2009, 48, 9797.

61 A. B. Gurav, Q. F. Xu, S. S. Latthe, R. S. Vhatkar, S. H. Liu, H. Yoon and S. S. Yoon, Ceram. Int., 2015, 41, 3017.

62 T. Zhu, C. Cai, C. T. Duan, S. Zhai, S. M. Liang, Y. Jin, N. Zhao and J. Xu, ACS Appl. Mater. Interfaces, 2015, 7, 13996.

63 L. Wu, J. Zhang, B. Li, L. Fan, L. Li and A. Wang, J. Colloid Interface Sci., 2014, 432, 31.

64 Y. K. Lai, X. F. Gao, H. F. Zhuang, J. Y. Huang, C. J. Lin and L. Jiang, Adv. Mater., 2009, 21, 3799.

65 Y. K. Lai, L. Sun, Y. C. Chen, H. F. Zhuang, C. J. Lin and J. W. Chin, J. Electrochem. Soc., 2006, 153, D123.

66 J. Y. Huang, Y. K. Lai, L. N. Wang, S. H. Li, M. Z. Ge, K. Q. Zhang, H. Fuchs and L. F. Chi, J. Mater. Chem. A, 2014, 2, 18531.

67 X. M. Bao, J. F. Cui, H. X. Sun, W. D. Liang, Z. Q. Zhu, J. An, B. P. Yang, P. Q. La and A. Li, Appl. Surf. Sci., 2014, 303, 473. 68 P. Aminayi and N. Abidi, Appl. Surf. Sci., 2013, 287, 223.

69 Z. R. Zheng, Z. Y. Gu, R. T. Huo and Y. H. Ye, Appl. Surf. Sci., 2009, 255, 7263.

70 B. Balu, V. Breedveld and D. W. Hess, Langmuir, 2008, 24, 4785.

71 J. Oh, T. Ko, M. Moon and C. H. Park, RSC Adv., 2014, 4, 38966.

72 D. Caschera, A. Mezzi, L. Cerri, T. de Caro, C. Riccucci, G. M. Ingo, G. Padeletti, M. Biasiucci, G. Gigli and B. Cortese, Cellulose, 2014, 21, 741.

73 D. Caschera, R. G. Toro, F. Federici, C. Riccucci, G. M. Ingo, G. Gigli and B. Cortese, Cellulose, 2015, 22, 2797.

74 D. Nyström, J. Lindqvist, E. Östmark, P. Antoni, A. Carlmark, A. Hult and E. Malmström, ACS Appl. Mater. Interfaces, 2009, 1, 816.

75 S. W. Li, T. L. Xing, Z. X. Li and G. Q. Chen, Appl. Surf. Sci., 2013, 268, 92.

76 J. D. Wu, Y. L. Jiang, D. J. Jiang, J. He, G. Q. Cai and J. P. Wang, Mater. Lett., 2015, 160, 384.

77 S. H. Li, J. Y. Huang, M. Z. Ge, S. W. Li, T. L. Xing, G. Q. Chen, Y. Q. Liu, K. Q. Zhang, S. S. Al-Deyab and Y. K. Lai, Mater. Des., 2015, 85, 815.

78 C. H. Xue, X. J. Guo, J. Z. Ma and S. T. Jia, ACS Appl. Mater. Interfaces, 2015, 7, 8251.

79 Y. Zhang, Y. Q. Li, J. Z. Shao and C. Zou, Surf. Coat. Technol., 2015, 276, 16.

80 Y. W. Li, X. W. Zheng, H. Y. Zhu, K. Wu and M. G. Lu, RSC Adv., 2015, 5, 46132.

81 B. Wang, J. Li, G. Y. Wang, W. X. Liang, Y. B. Zhang, L. Shi, Z. G. Guo and W. M. Liu, ACS Appl. Mater. Interfaces, 2013, 5, 1827. 
82 H. Zhou, H. X. Wang, H. T. Niu, J. Fang, Y. Zhao and T. Lin, Adv. Mater. Interfaces, 2015, 2, 1400559.

83 H. X. Wang, H. Zhou, A. Gestos, J. Fang, H. T. Niu, J. Ding and T. Lin, Soft Matter, 2013, 9, 277.

84 J. S. Wang and K. Matyjaszewski, Macromolecules, 1995, 28, 7901.

85 J. S. Wang and K. Matyjaszewski, J. Am. Chem. Soc., 1995, 117, 5614.

86 M. Kato, M. Kamigaito, M. Sawamoto and T. Higashimura, Macromolecules, 1995, 28, 1721.

87 M. Yin, W. D. Habicher and B. Voit, Polymer, 2005, 46, 3215.

88 L. N. Pilon, S. P. Armes, P. Findlay and S. P. Rannard, Eur. Polym. J., 2006, 42, 1487.

89 R. R. Shah, D. Merreceyes, M. Husemann, I. Rees, N. L. Abbott, C. J. Hawker and J. L. Hedrick, Macromolecules, 2000, 33, 597.

90 J. B. Kim, M. L. Bruening and G. L. Baker, J. Am. Chem. Soc., 2000, 122, 7616.

91 T. V. Werne and T. E. Patten, J. Am. Chem. Soc., 1999, 121, 7409.

92 B. Zhao and W. J. Brittain, J. Am. Chem. Soc., 1999, 121, 3557.

93 M. Husseman, E. E. Malmstrom, M. McNamara, M. Mate, D. Mecerreyes, D. G. Benoit, J. L. Hedrick, P. Mansky, E. Huang, T. P. Russell and C. J. Hawker, Macromolecules, 1999, 32, 1424.

94 M. Ejaz, S. Yamamoto, K. Ohno, Y. Tsujii and T. Fukuda, Macromolecules, 1998, 31, 5934.

95 T. Liu, R. Casado-Portilla, J. Belmont and K. Matyjaszewski, J. Polym. Sci., Part A: Polym. Chem., 2005, 43, 4695.

96 H. Kong, C. Gao and D. Yan, J. Am. Chem. Soc., 2004, 126, 412.

97 D. Plackett, K. Jankova, H. Egsgaard and S. Hvilsted, Biomacromolecules, 2005, 6, 2474.

98 A. Ramakrishnan, R. Dhamodharan and J. Ruhe, J. Polym. Sci., Part A: Polym. Chem., 2006, 44, 1758.

99 H. L. Zou, S. D. Lin, Y. Y. Tu, G. J. Liu, J. W. Hu, F. Li, L. Miao, G. W. Zhang, H. S. Luo, F. Liu, C. M. Hou and M. L. Hu, J. Mater. Chem. A, 2013, 1, 11246.

100 H. L. Zou, S. D. Lin, Y. Y. Tu, F. Li, J. W. Hu, G. J. Liu, S. Y. Hu, G. H. Yang and Z. W. Yu, Adv. Mater. Interfaces, 2016, 3, 1500693.

101 S. Nagappan, S. S. Park and C. S. Ha, J. Nanosci. Nanotechnol., 2014, 14, 1441.

102 G. W. Zhang, J. W. Hu, Y. Y. Tu, G. P. He, F. Li, H. L. Zou, S. D. Lin and G. H. Yang, Phys. Chem. Chem. Phys., 2015, 17, 19457.

103 G. W. Zhang, S. D. Lin, I. Wyman, H. L. Zou, J. W. Hu, G. J. Liu, J. D. Wang, F. Li, F. Liu and M. L. Hu, ACS Appl. Mater. Interfaces, 2013, 5, 13466.

104 F. Li, Y. Y. Tu, J. W. Hu, H. L. Zou, G. J. Liu, S. D. Lin, G. H. Yang, S. Y. Hu, L. Miao and Y. M. Moa, Polym. Chem., 2015, 6, 6746.

105 X. Yao, Y. Song and L. Jiang, Adv. Mater., 2011, 23, 719.

106 S. Wang, K. Liu, X. Yao and L. Jiang, Chem. Rev., 2015, 115, 8230 .

107 Y. Tian, B. Su and L. Jiang, Adv. Mater., 2014, 26, 6872.
108 J. P. Zhang and S. Seeger, Angew. Chem., Int. Ed., 2011, 50, 6652.

109 G. R. J. Artus, J. Zimmermann, F. A. Reifler, S. A. Brewer and S. Seeger, Appl. Surf. Sci., 2012, 258, 3835.

110 B. X. Leng, Z. Z. Shao, G. de With and W. H. Ming, Langmuir, 2009, 25, 2456.

111 H. X. Wang, Y. H. Xue and T. Lin, Soft Matter, 2011, 7, 8158.

112 H. X. Wang, Y. H. Xue, J. Ding, L. F. Feng, X. G. Wang and T. Lin, Angew. Chem., Int. Ed., 2011, 50, 11433.

113 H. Zhou, H. X. Wang, H. T. Niu, A. Gestos and T. Lin, Adv. Funct. Mater., 2013, 23, 1664.

114 Z. G. Xu, Y. Zhao, H. X. Wang, X. G. Wang and T. Lin, Angew. Chem., Int. Ed., 2015, 127, 4610.

115 V. A. Ganesh, S. S. Dinachali, H. K. Raut, T. M. Walsh, A. S. Nair and S. Ramakrishna, RSC Adv., 2013, 3, 3819.

116 J. Lin, C. Zheng, W. J. Ye, H. Q. Wang, D. Y. Feng, Q. Y. Li and B. W. Huan, J. Appl. Polym. Sci., 2015, 132, 41458.

117 Y. Lu, S. Sathasivam, J. L. Song, C. R. Crick, C. J. Carmalt and I. P. Parkin, Science, 2015, 347, 113.

118 W. F. Zhang, X. Lu, Z. Xin and C. L. Zhou, Nanoscale, 2015, 7, 19476.

119 M. Yu, Z. Q. Wang, H. Z. Liu, S. Y. Xie, J. X. Wu, H. Q. Jiang, J. Y. Zhang, L. F. Li and J. Y. Li, ACS Appl. Mater. Interfaces, 2013, 5, 3697.

120 J. Manna, S. Goswami, N. Shilpa, N. Sahu and R. K. Rana, ACS Appl. Mater. Interfaces, 2015, 7, 8076.

121 A. B. D. Cassie and S. Baxter, Trans. Faraday Soc., 1944, 40, 546.

122 R. N. Wenzel, Ind. Eng. Chem., 1936, 28, 988.

123 Y. X. Jin, Q. P. Ke, P. Jiang, Y. S. N. Zhu, F. H. Cheng and Y. X. Zhang, Appl. Surf. Sci., 2015, 351, 358.

124 C. T. Hsieh, J. P. Hsu, H. H. Hsu, W. H. Lin and R. S. Juang, Surf. Coat. Technol., 2016, 286, 148.

125 S. Xiong, L. Kong, J. Huang, X. Q. Chen and Y. Wang, J. Membr. Sci., 2015, 493, 478.

126 C. L. Zhang, P. Li and B. Cao, Ind. Eng. Chem. Res., 2016, 55, 5030.

127 Y. Oikawa, T. Saito, S. Yamada, M. Sugiya and H. Sawada, ACS Appl. Mater. Interfaces, 2015, 7, 13782.

128 Z. G. Xu, Y. Zhao, H. X. Wang, H. Zhou, C. X. Qin, X. G. Wang and T. Lin, ACS Appl. Mater. Interfaces, 2016, 8, 5661 .

129 M. Rana, B. Hao, L. Mu, L. Chen and P. C. Ma, Compos. Sci. Technol., 2016, 122, 104.

130 X. Y. Zhou, Z. Z. Zhang, X. H. Xu, F. Guo, X. T. Zhu, X. H. Men and B. Ge, ACS Appl. Mater. Interfaces, 2013, 5, 7208.

131 K. Lee, J. Hwang and Y. Ahn, Bull. Korean Chem. Soc., 2014, 35, 1545.

132 J. Li, L. Yan, Y. Z. Zhao, F. Zha, Q. T. Wang and Z. Q. Lei, Phys. Chem. Chem. Phys., 2015, 17, 6451.

133 Z. Y. Deng, W. Wang, L. H. Mao, C. F. Wang and S. Chen, J. Mater. Chem. A, 2014, 2, 4178.

134 Z. X. Wang, Y. C. Xu, Y. Y. Liu and L. Shao, J. Mater. Chem. A, 2015, 3, 12171.

135 J. T. Wang and Y. H. Chen, J. Appl. Polym. Sci., 2015, 132, 42614. 
136 X. T. Zhu, Z. Z. Zhang, B. Ge, X. H. Men, X. Y. Zhou and Q. J. Xue, J. Colloid Interface Sci., 2014, 432, 105.

137 Y. Li, J. D. Wang, L. N. Fan and D. R. Chen, Acta Phys.-Chim. Sin., 2016, 32, 990.

138 X. J. Liu, L. Ge, W. Li, X. Z. Wang and F. Li, ACS Appl. Mater. Interfaces, 2015, 7, 791.

139 S. J. Pan, R. Guo and W. J. Xu, AIChE J., 2014, 60, 2752.

140 Z. J. Wang, Y. Wang and G. J. Liu, Angew. Chem., 2016, 128, 1313.

141 C. H. Xue, Y. R. Li, J. L. Hou, L. Zhang, J. Z. Ma and S. T. Jia, J. Mater. Chem. A, 2015, 3, 10248.

142 F. Liu, M. L. Ma, D. L. Zang, Z. X. Gao and C. Y. Wang, Carbohydr. Polym., 2014, 103, 480.

143 Q. L. Ma, H. F. Cheng, A. G. Fane, R. Wang and H. Zhang, Small, 2016, 12, 2186.

144 X. Zheng, Z. Y. Guo, D. L. Tian, X. F. Zhang, W. X. Li and L. Jiang, ACS Appl. Mater. Interfaces, 2015, 7, 4336.

145 J. Li, L. Yan, X. H. Tang, H. Feng, D. C. Hu and F. Zha, Adv. Mater. Interfaces, 2016, 3, 1500770.

146 Y. Y. Liu, J. H. Xin and C. H. Choi, Langmuir, 2012, 28, 17426.

147 H. Zhou, H. X. Wang, H. T. Niu and T. Lin, Sci. Rep., 2013, 3, 2964.

148 S. Kwon, T. J. Ko, E. Yu, M. W. Moon and C. H. Park, RSC Adv., 2014, 4, 45442.

149 K. Sasaki, M. Tenjimbayashi, K. Manabe and S. Shiratori, ACS Appl. Mater. Interfaces, 2016, 8, 651.

150 X. L. Tian, H. Jin, J. Sainio, R. H. A. Ras and O. Ikkala, Adv. Funct. Mater., 2014, 24, 6023.

151 Y. Wang, X. Y. Li, H. Hu, G. J. Liu and M. Rabnawaz, J. Mater. Chem. A, 2014, 2, 8094.

152 Y. Liu, X. W. Wang, B. Fei, H. W. Hu, C. L. Lai and J. H. Xin, Adv. Funct. Mater., 2015, 25, 5047.

153 S. Li, S. Zhang and X. Wang, Langmuir, 2008, 24, 5585.

154 R. Dastjerdi, M. Montazer and S. Shahsavan, Colloids Surf., $B, 2010,81,32$.

155 B. Deng, R. Cai, Y. Yu, H. Q. Jiang, C. L. Wang, J. Li, L. F. Li, M. Yu, J. Y. Li, L. D. Xie, Q. Huang and C. H. Fan, Adv. Mater., 2010, 22, 5473.

156 R. Abbas, M. A. Khereby, W. A. Sadik and A. G. M. El Demerdash, Cellulose, 2015, 22, 887.

157 J. Zimmermann, F. A. Reifler, G. Fortunato, L. C. Gerhardt and S. Seeger, Adv. Funct. Mater., 2008, 18, 3662.

158 H. Zhou, H. X. Wang, H. T. Niu, A. Gestos, X. G. Wang and T. Lin, Adv. Mater., 2012, 24, 2409.

159 C. H. Xue, P. Zhang, J. Z. Ma, P. T. Ji, Y. R. Li and S. T. Jia, Chem. Commun., 2013, 49, 358.

160 M. E. Yazdanshenas and M. Shateri-Khalilabad, Ind. Eng. Chem. Res., 2013, 52, 12846.

161 I. Das and G. De, Sci. Rep., 2015, 5, 18503.

162 C. H. Xue, Y. R. Li, P. Zhang, J. Z. Ma and S. T. Jia, ACS Appl. Mater. Interfaces, 2014, 6, 10153.

163 M. C. Wu, B. H. Ma, T. Z. Pan, S. S. Chen and J. Q. Sun, Adv. Funct. Mater., 2016, 26, 569.

164 G. H. Xi, J. Wang, G. Y. Luo, Y. H. Zhu, W. C. Fan, M. Q. Huang, H. Q. Wang and X. D. Liu, Cellulose, 2016, 23, 915.
165 S. S. Chen, X. Li, Y. Li and J. Q. Sun, ACS Nano, 2015, 9, 4070 .

166 Y. H. Liu, Z. L. Liu, Y. P. Liu, H. Y. Hu, Y. Li, P. X. Yan, B. Yu and F. Zhou, Small, 2015, 4, 426.

167 C. Shillingford, N. MacCallum, T. S. Wong, P. Kim and J. Aizenberg, Nanotechnology, 2014, 25, 014019.

168 Y. Li, B. Ge, X. H. Men, Z. Z. Zhang and Q. J. Xue, Compos. Sci. Technol., 2016, 125, 55.

169 J. X. Wu, J. Y. Li, B. Deng, H. Q. Jiang, Z. Q. Wang, M. Yu, L. F. Li, C. Y. Xing and Y. J. Li, Sci. Rep., 2013, 3, 2951.

170 Y. F. Wang, X. W. Wang, C. L. Lai, H. W. Hu, Y. Kong, B. Fei and J. H. Xin, ACS Appl. Mater. Interfaces, 2016, 8, 2950.

171 L. H. Zou, C. T. Lan, X. P. Li, S. L. Zhang, Y. P. Qiu and Y. Ma, Fibers Polym., 2015, 10, 2158.

172 Y. He, C. Y. Jiang, X. B. Cao, J. Chen, W. Tian and W. Z. Yuan, Appl. Surf. Sci., 2014, 305, 589.

173 A. Davis, Y. H. Yeong, A. Steele, I. S. Bayer and E. Loth, ACS Appl. Mater. Interfaces, 2014, 6, 9272.

174 A. Dotan, H. Dodiuk, C. Laforte and S. J. Kenig, J. Adhes. Sci. Technol., 2009, 23, 1907.

175 J. Y. Lv, Y. L. Song, L. Jiang and J. J. Wang, ACS Nano, 2014, 8, 3152.

176 H. Zhu, Z. G. Guo and W. M. Liu, Chem. Commun., 2014, 50, 3900.

177 Y. Y. Wang, J. Xue, Q. J. Wang, Q. M. Chen and J. F. Ding, ACS Appl. Mater. Interfaces, 2013, 5, 3370.

178 S. L. Zheng, C. Li, Q. T. Fu, T. F. Xiang, W. Hu, J. Wang, S. B. Ding, P. J. Liu and Z. Chen, RSC Adv., 2016, 6, 79389.

179 M. A. Sarshar, C. Swarctz, S. Hunter, J. Simpson and C. H. Choi, Colloid Polym. Sci., 2013, 291, 427.

180 F. Arianpour, M. Farzaneh and S. A. Kulinich, Appl. Surf. Sci., 2013, 265, 546.

181 S. L. Zheng, C. Li, Q. T. Fu, W. Hu, T. F. Xiang, Q. Wang, M. P. Du, X. C. Liu and Z. Chen, Mater. Des., 2016, 93, 261.

182 Y. Z. Shen, J. Tao, H. J. Tao, S. L. Chen, L. Pan and T. Wang, Langmuir, 2015, 31, 10799.

183 Y. Z. Shen, J. Tao, H. J. Tao, S. L. Chen, L. Pan and T. Wang, RSC Adv., 2015, 5, 32813.

184 Y. Z. Shen, H. J. Tao, S. L. Chen, L. M. Zhu, T. Wang and J. Tao, RSC Adv., 2015, 5, 1666.

185 J. Chen, Z. Q. Luo, Q. R. Fan, J. Y. Lv and J. J. Wang, Small, 2014, 10, 4693.

186 R. M. Dou, J. Chen, Y. F. Zhang, X. P. Wang, D. P. Cui, Y. L. Song, L. Jiang and J. J. Wang, ACS Appl. Mater. Interfaces, 2014, 6, 6998.

187 Y. L. Wang, X. Yao, J. Chen, Z. Y. He, J. Liu, Q. Y. Li, J. J. Wang and L. Jiang, Sci. China Mater., 2015, 58, 559.

188 L. Mishchenko, B. Hatton, V. Bahadur, J. A. Taylor, T. Krupenkin and J. Aizenberg, ACS Nano, 2010, 4, 7699.

189 X. Yao, Y. Song and L. Jiang, Adv. Mater., 2011, 23, 719.

190 J. Xiao and S. Chaudhuri, Langmuir, 2012, 28, 4434.

191 J. Liu, H. Y. Guo, B. Zhang, S. S. Qiao, M. Z. Shao, X. R. Zhang, X. Q. Feng, Q. Y. Li, Y. L. Song, L. Jiang and J. J. Wang, Angew. Chem., Int. Ed., 2016, 55, 4265.

192 N. Dalili, A. Edrisy and R. Carriveau, Renewable Sustainable Energy Rev., 2009, 13, 428.

193 O. Parent and A. Ilinca, Cold Reg. Sci. Technol., 2011, 65, 88. 
194 L. Cao, A. K. Jones, V. K. Sikka, J. Wu and D. Gao, Langmuir, 2009, 25, 12444.

195 A. J. Meuler, G. H. McKinley and R. E. Cohen, ACS Nano, 2010, 4, 7048.

196 L. B. Boinovich, A. M. Emelyanenko, V. K. Ivanov and A. S. Pashinin, ACS Appl. Mater. Interfaces, 2013, 5, 2549.

197 H. J. Lee, J. Mater. Sci., 2012, 47, 5114.

198 Y. Y. Zhang, Z. L. Jiang, J. Y. Huang, L. Y. Lim, W. L. Li, J. Y. Deng, D. G. Gong, Y. X. Tang, Y. K. Lai and Z. Chen, RSC Adv., 2015, 5, 79479.

199 B. Su, Y. Tian and L. Jiang, J. Am. Chem. Soc., 2016, 138, 1727.

200 X. Deng, L. Mammen, H. J. Butt and D. Vollmer, Science, 2012, 335, 67.

201 A. Tuteja, W. Choi, M. L. Ma, J. M. Mabry, S. A. Mazzella, G. C. Rutledge, G. H. McKinley and R. E. Cohen, Science, 2007, 318, 1618.

202 C. De Marco, V. Oldani, C. L. Bianchi, M. Levi and S. Turri, J. Appl. Polym. Sci., 2015, 132, 42404.

203 H. X. Wang, J. Fang, T. Cheng, J. Ding, L. T. Qu, L. M. Dai, X. G. Wang and T. Lin, Chem. Commun., 2008, 877.

204 C. Y. Cao, M. Z. Ge, J. Y. Huang, S. H. Li, S. Deng, S. N. Zhang, Z. Chen, K. Q. Zhang, S. S. Al-Deyab and Y. K. Lai, J. Mater. Chem. A, 2016, 4, 12179.

205 Y. K. Lai, J. Y. Huang, Z. Q. Cui, M. Z. Ge, K. Q. Zhang, Z. Chen and L. F. Chi, Small, 2016, 12, 2203.

206 J. Y. Huang, Y. K. Lai, F. Pan, L. Yang, H. Wang, K. Q. Zhang, H. Fuchs and L. F. Chi, Small, 2014, 10, 4865.

207 H. Liu, S. W. Gao, J. S. Cai, C. L. He, J. J. Mao, T. X. Zhu, Z. Chen, J. Y. Huang, K. Meng, K. Q. Zhang, S. S. Al-Deyab and Y. K. Lai, Materials, 2016, 9, 124.
208 X. F. Wang, B. Ding, G. Sun, M. R. Wang and J. Y. Yu, Prog. Mater. Sci., 2013, 58, 1173.

209 Y. Si, Q. X. Fu, X. Q. Wang, J. Zhu, J. Y. Yu, G. Sun and B. Ding, ACS Nano, 2015, 9, 3791.

210 L. P. Wen, Y. Tian and L. Jiang, Angew. Chem., Int. Ed., 2015, 54, 3387.

211 T. Darmanin and F. Guittard, J. Mater. Chem. A, 2014, 2, 16319.

212 H. Bellanger, T. Darmanin, E. T. de Givenchy and F. Guittard, Chem. Rev., 2014, 114, 2694.

213 S. N. Zhang, J. Y. Huang, Y. X. Tang, S. H. Li, M. Z. Ge, Z. Chen, K. Q. Zhang and Y. K. Lai, Small, 2016, 12, DOI: 10.1002/smll.201600687.

214 Y. K. Lai, F. Pan, C. Xu, H. Fuchs and L. F. Chi, Adv. Mater., 2013, 25, 1682.

215 Y. K. Lai, L. X. Lin, F. Pan, J. Y. Huang, R. Song, Y. X. Huang, C. J. Lin, H. Fuchs and L. F. Chi, Small, 2013, 9, 2945.

216 H. Q. Li, Y. K. Lai, J. Y. Huang, Y. X. Tang, L. Yang, Z. Chen, K. Q. Zhang, X. C. Wang and L. P. Tan, J. Mater. Chem. B, 2015, 3, 342 .

217 Y. Zhao, H. X. Wang, H. Zhou and T. Lin, Small, 2016, 12, DOI: $10.1002 /$ smll.201601070.

218 Y. J. Lei, R. Z. Sun, X. C. Zhang, X. J. Feng and L. Jiang, Adv. Mater., 2016, 28, 1477.

219 C. L. Hao, Y. H. Liu, X. M. Chen, J. Li, M. Zhang, Y. H. Zhao and Z. K. Wang, Small, 2016, 12, 1825.

220 Y. H. Liu, L. Moevius, X. P. Xu, T. Z. Qian, J. M. Yeomans and Z. K. Wang, Nat. Phys., 2014, 10, 515. 